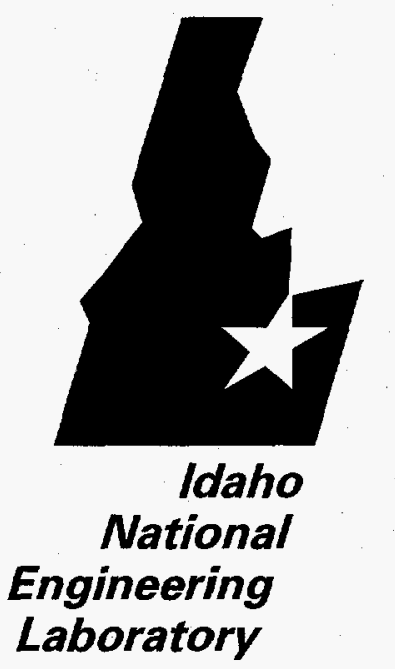

INEL/EXT-97-00178

March 1997

\title{
Lift Truck Safety Review
}

\section{RECEIVED \\ JUN $O 91997$ \\ OSTI}

L. C. Cadwallader

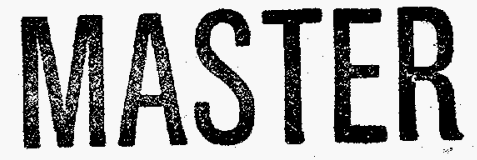

LOCKHEED MARTIN 


\section{DISCLAIMER}

This report was prepared as an account of work sponsored by an agency of the United States Government. Neither the United States Government nor any agency thereof, nor any of their employees, makes any warranty, express or implied, or assumes any legal liability or responsibility for the accuracy, completeness, or usefulness of any information, apparatus, product or process disclosed, or represents that its use would not infringe privately owned rights. References herein to any specific commercial product, process, or service by trade name, trademark, manufacturer, or othenwise, does not necessarily constitute or imply its endorsement, recommendation, or favoring by the United States Government or any agency thereof. The views and opinions of authors expressed herein do not necessarily state or reflect those of the United States Government or any agency thereot. 


\section{DISCLAIMER}

This report was prepared as an account of work sponsored by an agency of the United States Government. Neither the United States Government nor any agency thereof, nor any of their employees, make any warranty, express or implied, or assumes any legal liability or responsibility for the accuracy, completeness, or usefulness of any information, apparatus, product, or process disclosed, or represents that its use would not infringe privately owned rights. Reference herein to any specific commercial product, process, or service by trade name, trademark, manufacturer, or otherwise does not necessarily constitute or imply its endorsement, recommendation, or favoring by the United States Government or any agency thereof. The views and opinions of authors expressed herein do not necessarily state or reflect those of the United States Government or any agency thereof. 


\section{DISCLAMMER}

Portions of this document may be illegible in electronic image products. Images are produced from the best available original document. 


\section{Lift Truck Safety Review}

L. C. Cadwallader

Published March 1997

Idaho National Engineering and Environmental Laboratory

Nuclear Engineering Technologies Department

Lockheed Martin Idaho Technologies Company

Idaho Falls, ID 83415-3815

Prepared for the

U.S. Department of Energy

Office of Energy Research

Under DOE Field Office, Idaho

Contract No. DE-AC07-941D13223 


\begin{abstract}
This report presents safety information about powered industrial trucks. The basic lift truck, the counterbalanced sit down rider truck, is the primary focus of the report. Lift truck engineering is briefly described, then a hazard analysis is performed on the lift truck. Case histories and accident statistics are also given. Rules and regulations about lift trucks, such as the US Occupational Safety and Health Administration laws and the Underwriter's Laboratories standards, are discussed. Safety issues with lift trucks are reviewed, and lift truck safety and reliability are discussed. Some quantitative reliability values are given.
\end{abstract}




\section{Summary}

Department of Energy facilities undergo all phases of the facility life cycle, from construction, to operation, and then decommissioning. In an effort to look deeper at operational safety, this study has reviewed powered industrial truck safety information because these vehicles are used throughout the facility life cycle, and US industry has suffered many injury and fatality events related to these trucks.

This report is a collection of safety-related information about powered industrial trucks used for moving materials in energy facilities, industrial plants, and waste storage centers. One facet of operational safety is the ability to assess industrial hazards as well as radiological and toxicological hazards. The powered industrial truck, or lift truck, has been a great asset to materials handling, but it has also created new workplace injuries and is also responsible for about 100 workplace fatalities each year in the US. Material losses from lift truck related accidents were over $\$ 160$ million in the US between 1987-1992. To enjoy the productivity benefits of the lift truck, its liabilities must be well managed.

Managing the lift truck hazard begins with understanding how a lift truck operates. Lift truck systems are briefly described. The system operating conditions of temperature, pressure, and any other pertinent parameters are discussed. Next, the hazards that the lift truck introduces into the workplace are defined. An analysis of energy sources on the lift truck is used to identify hazards. Operating experiences from the literature are summarized, and safety literature about lift trucks has been reviewed to identify any other concerns that might be present with this piece of equipment.

Regulations, rules, consensus standards, recommended practices, and guidance about lift truck safety was collected from the literature and has been summarized. There is a large amount of information available. Most of the literature is not legally binding, but those parts that are binding do impose a great responsibility onto lift truck owners and operators. Daily inspections for mechanical and electrical readiness, removal from operation for almost any technical problem, operator training, and rules of operation are all given as regulations.

Some safety issues of interest with lift trucks are discussed, including the use of seat belts and operator training programs. There has been a preoccupation with lift truck fire safety, and this issue is also discussed.

Recent safety analyses that have included lift trucks, because of the sensitive cargo that they carry, have been summarized. This summary forms guidance of its own to support analysts in performing future lift truck safety analyses for public safety, operational safety of a process, and the safety of personnel using, or in proximity of, the lift truck. Some reliability issues, including some quantitative failure rate information has been presented. These data are sparse; therefore, having some data to report may be useful for facility operations personnel and maintenance personnel. 


\section{Table of Contents}

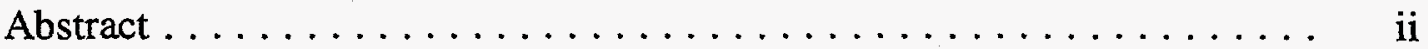

Summary $\ldots \ldots \ldots \ldots \ldots \ldots \ldots \ldots \ldots \ldots \ldots \ldots \ldots \ldots \ldots \ldots$ iii

Nomenclature $\ldots \ldots \ldots \ldots \ldots \ldots \ldots \ldots \ldots \ldots \ldots \ldots \ldots \ldots \ldots$

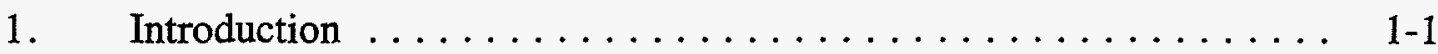

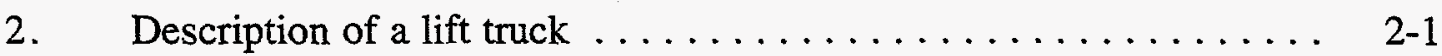

3. Safety issues with lift trucks $\ldots \ldots \ldots \ldots \ldots \ldots \ldots \ldots \ldots \ldots \ldots \ldots, 1$

Hazards analysis . . . . . . . . .

Operating experiences $\ldots \ldots \ldots \ldots \ldots \ldots \ldots, 3-3$

Literature review of lift truck hazards $\ldots \ldots \ldots \ldots \ldots \ldots, 3-20$

Conclusions $\ldots \ldots \ldots \ldots \ldots \ldots \ldots \ldots \ldots \ldots, 3-21$

4. Regulations and standards for lift trucks $\ldots \ldots \ldots \ldots \ldots \ldots \ldots$ 4-1

Code of Federal Regulations ................ 4-1

Underwriters Laboratories . . . . . . . . . . . . . . 4-5

National Fire Protection Association $\ldots \ldots \ldots \ldots \ldots \ldots$ 4 4-6

American National Standards Institute $\ldots \ldots \ldots \ldots \ldots \ldots$ 4. 4

Society of Automotive Engineers $\ldots \ldots \ldots \ldots \ldots \ldots$. 4-9

Factory Mutual ..................... 4-10

National Safety Council ................... 4-11

US Department of Energy $\ldots \ldots \ldots \ldots \ldots \ldots \ldots \ldots$ 4-11

Conclusions $\ldots \ldots \ldots \ldots \ldots \ldots \ldots \ldots \ldots, 4-12$

5. Current issues and concerns for lift truck safety ........... $5-1$

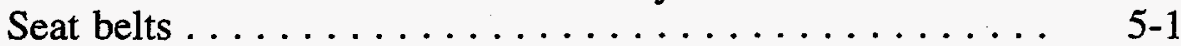

Fire extinguishers $\ldots \ldots \ldots \ldots \ldots \ldots \ldots \ldots \ldots, 5-1$

Engine fire suppression system $\ldots \ldots \ldots \ldots \ldots \ldots \ldots, 5-1$

Driver visibility $\ldots \ldots \ldots \ldots \ldots \ldots \ldots \ldots \ldots \ldots, 5-2$

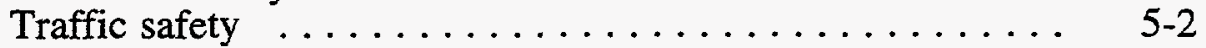

Operator training $\ldots \ldots \ldots \ldots \ldots \ldots \ldots \ldots \ldots \ldots, 5-3$

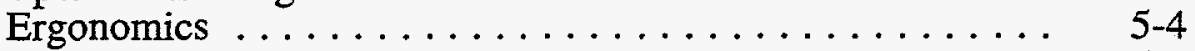

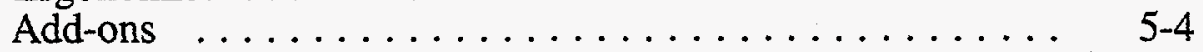

6. Lift truck safety analysis and reliability $\ldots \ldots \ldots \ldots \ldots \ldots \ldots, 6-1$

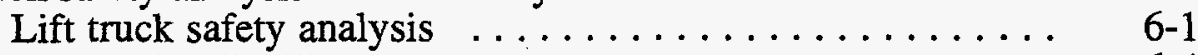

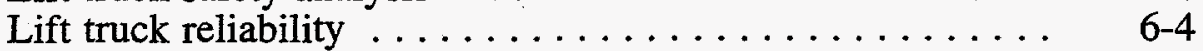

Conclusions $\ldots \ldots \ldots \ldots \ldots \ldots \ldots \ldots \ldots \ldots$ 6-6 


\section{Nomenclature}

\begin{tabular}{|c|c|}
\hline $\begin{array}{l}\text { ANSI } \\
\text { ASME } \\
{ }^{\circ} \mathrm{C} \\
\text { CFR } \\
\mathrm{cm}\end{array}$ & $\begin{array}{l}\text { American National Standards Institute } \\
\text { American Society of Mechanical Engineers } \\
\text { degrees Celsius } \\
\text { Code of Federal Regulations } \\
\text { centimeter }\end{array}$ \\
\hline $\begin{array}{l}\text { CNG } \\
\mathrm{dB} \\
\mathrm{DOE} \\
{ }^{\circ} \mathrm{F} \\
\mathrm{FM}\end{array}$ & $\begin{array}{l}\text { compressed natural gas } \\
\text { decibels } \\
\text { Department of Energy } \\
\text { degrees Fahrenheit } \\
\text { Factory Mutual }\end{array}$ \\
\hline $\begin{array}{l}\text { FOPS } \\
\text { fps } \\
\text { FR } \\
\text { gpm } \\
\mathrm{kPa}\end{array}$ & $\begin{array}{l}\text { Falling Object Protective Structure } \\
\text { feet per second } \\
\text { Federal Register } \\
\text { gallons per minute } \\
\text { kilopascals }\end{array}$ \\
\hline $\begin{array}{l}\mathrm{kph} \\
\mathrm{LP} \\
\mathrm{m} \\
\mathrm{mm} \\
\mathrm{mph}\end{array}$ & $\begin{array}{l}\text { kilometers per hour } \\
\text { liquefied petroleum gas } \\
\text { meters } \\
\text { millimeters } \\
\text { miles per hour }\end{array}$ \\
\hline $\begin{array}{l}\text { NFPA } \\
\text { NSC } \\
\text { OSHA } \\
\text { psig } \\
\text { ROPS } \\
\text { SAE }\end{array}$ & $\begin{array}{l}\text { National Fire Protection Association } \\
\text { National Safety Council } \\
\text { Occupational Safety and Health Administration } \\
\text { pounds per square inch, gauge } \\
\text { Roll Over Protective Structure } \\
\text { Society of Automotive Engineers }\end{array}$ \\
\hline
\end{tabular}




\section{LIFT TRUCK SAFETY REVIEW}

\section{Introduction}

The powered industrial truck, also called lift truck or forklift, is a device for moving large amounts of material quickly and efficiently. Lift trucks are effective for lifting, stacking, and unloading materials from storage racks, road trucks, rail cars, and other equipment. Lift trucks move unit quantities to varying destinations; this design is more flexible than using other material handling methods (conveyor belts, etc.). Unit quantities might be drums, boxes, sacks, etc. Pallets made of wood, cardboard, or plastic (FM, 1992) are used as a means to provide height to allow forks underneath the load for lifting. The pallets remain under the load when it reaches its destination. Pallets are inexpensive and are considered to be expendable.

The lift truck has often been cited as having been invented during World War II (for example, see Brannigan, 1992), but early versions have been in use since the turn of the century (Bowman, 1972). The most common of all lift trucks is the sit down rider truck, counterbalanced to carry a load (Hodson, 1992). Other types of lift trucks are the stand up rider truck for narrow aisle applications, straddle trucks that carry a load amidships, and non-rider trucks such as motorized pallet jacks. A brief description of the sit down rider lift truck is given in Chapter 2. While automated vehicles have been used in some factory situations, the lift truck is still a workhorse of industry (Holzhauer, 1990).

Lift trucks have aided industry by replacing people in repetitive material handling operations. The potential for injuries by having people manually move loads, such as drums of liquid waste or construction materials, is large. Using lift trucks has increased the speed and weights of flowing materials while freeing people from manual material handling injuries. Many industries use lift trucks; Department of Energy facilities have use for lift trucks during construction, operations, and decommissioning activities. In construction, rough terrain outdoor lift trucks can move construction materials - beams, pipe sections, pallets of building materials, equipment items (fans, pumps, compressors, etc.), shielding materials, and other items. During operations, indoor lift trucks move drums of industrial materials (oil for large motors and engines, demineralizer resins, radioactive and chemical wastes, etc.) and pallets of consumable materials to and from road trucks. Lift trucks and cranes also move plant equipment for maintenance or refurbishment. In decommissioning activities, lift trucks move equipment items for decontamination and decommissioning, and they move boxes or casks of waste to the flat bed trailers of road trucks for shipment to burial grounds. The safety of lift trucks is an important aspect of facility operational safety in all phases of facility lifetime.

While lift trucks are quite useful and beneficial, they have also introduced new hazards into the workplace. Each year, approximately 100 U.S. workers are killed in forklift related incidents, and in 1992 there were 18,200 forklift related injuries that required days away from work (NSC, 1995). If the benefits of lift trucks are to be enjoyed, their liabilities must be managed to preclude or reduce the likelihood of an accident where workers could be injured or killed. Despite the provisions in the Occupational Safety and Health Act of 
1970 , lift trucks still claim a relatively constant number of fatalities each year ( $2 \%$ of total workplace deaths; see NSC, 1995). This report addresses the safety issues concerning lift trucks, and operations issues - reliability, availability, maintainability, and accident frequencies. The report chapters contain a lift truck description, a hazards assessment, a summary of lift truck regulations and standards, discussion of selected safety-related questions, and a discussion of safety analysis and reliability issues.

\section{Chapter 1 References}

Bowman, 1972. D. Bowman, Lift Trucks, A Practical Guide for Buyers and Users, Cahners Books, Boston, Massachusetts, ISBN 0-8436-1007-7, 1972.

Brannigan, 1992. F. L. Brannigan, Building Construction for the Fire Service, National Fire Protection Association, Quincy, Massachusetts, ISBN 9-87765-381-X, 1992, page 606.

FM, 1992.

"New Protection Criteria and a Better Plastic Pallet," Factory Mutual Record. the Magazine of Property Conservation, 69 , November/ December 1992, pages 9-12.

Hodson, 1992. W. K. Hodson, editor, Maynard's Industrial Engineering Handbook, fourth edition, McGraw-Hill, Inc., New York, ISBN 007-041086-0, 1992, chapter 13.

Holzhauer, 1990. R. Holzhauer, "Lift Truck Specifications Chart," Plant Engineering, 44, February 8, 1990, pages 65-85.

NSC, 1995.

National Safety Council, Accident Facts, 1995 edition, NSC, Itasca, Illinois, ISBN 0-87912-185-8, 1995. 


\section{Description of a Lift Truck}

The lift truck in common use is the sit down rider, counterbalanced truck (Hodson, 1992). This type of lift truck lifts pallet loads outside of the framework of the truck, using the truck's weight to balance the load. The front wheels (the drive wheels) act as a fulcrum. This is an important point to note later when discussing truck stability. Lift trucks can weigh a great deal (in the 1,800 to 4,500 kilogram [ 4,000 to 10,000 pound] range), since the truck itself must balance the load it carries. Most lift trucks are shorter in length than automobiles, but weigh up to twice the weight of a typical automobile.

There are several important subsystems on a lift truck (Bowman, 1972). These are the engine and its fuel system, the exhaust system, the forks and the hydraulic systems for controlling the load, and brakes. Each of these systems is discussed to provide insights into the energies used in lift truck operation.

Lift trucks can have two basic engine types, an electric motor engine or an internal combustion engine (Bowman, 1972). The internal combustion engine can run on either gasoline, diesel fuel, Liquefied Petroleum (LP) gas or gaseous propane fuel, or compressed natural gas $(\mathrm{CNG})$ fuel. Each type of engine has its own advantages and disadvantages. The electric motor is quiet, has no exhaust emissions, has little vibration, can be a low maintenance unit, has inherent braking (the motor provides braking resistance when the truck inertia is driving the motor), can have longevity compared to other engines (Holzhauer, 1992), and does not greatly warm up the air in the working area. However, the electric motor batteries require daily recharging times of several hours, these trucks are usually rated for slower speeds (usually $16 \mathrm{kph}$ [10 mph] maximum), lesser climbing ability, and lesser capacity than internal combustion engine trucks (Holzhauer, 1997). The internal combustion powered lift trucks are loud, have exhaust emissions that are a health concern in poorly ventilated areas, have high vibration, require frequent maintenance tune ups for engine efficiency and cleaner burn of fuel, and these trucks can warm up the air in the work area. Internal combustion engines generally give more power, so the trucks can climb steep slopes (up to $25^{\circ}$ inclines), can handle larger loads, and can travel at speeds over $16 \mathrm{kph}(10 \mathrm{mph})$. The engine type is left to the discretion of the plant engineer to choose the most suitable type by taking economics, efficiency, and safety into account. Transmissions and gear trains that complete the power train of the lift truck are very similar for internal combustion engines. The electric motor trucks vary because the direct current motor fits directly onto the drive axle, but these lift trucks can also use an automatic transmission.

Internal combustion trucks must have fuel systems and exhaust systems. Battery powered lift trucks also have concerns about battery leakage just as liquid or gaseous fuel systems have leakage concerns. Fuel tank sizes for internal combustion engine trucks can be 38 to 380 liters (10 to 100 gallons), depending on the size of the truck. The internal combustion engine exhaust system must stop sparks, backfire flames, and hot particulates (combustion products) from entering the facility environment as a safety measure to prevent ignition of fires and explosions. Some older lift trucks use water in the mufflers to quench hot particles and sparks, and to reduce exhaust temperatures. More modern lift trucks use 
baffle mufflers similar to automobile mufflers. Lift trucks for hazardous locations use flame arrestors in the exhaust system to preclude any sparks or flames from backfires emerging and igniting combustible gases, dusts, or fibers (Oliver, 1989). Electric powered trucks do not have exhaust systems.

The two forks face forward and are attached to a mast which can tilt forward or backward up to $10^{\circ}$ (or even 20 to $30^{\circ}$, depending on the model of truck) from vertical to unload cargo and to steady cargo while the lift truck is in motion. The mast tilt is usually accomplished with a hydraulic piston system, and raising the forks up and down the mast is also accomplished with hydraulic power provided by a hydraulic pump. Hydraulic system pressures can be as high as 7 to $21 \mathrm{MPa}$ (1000 to $3000 \mathrm{psig}$ ). The piston for vertical travel (the lift tube) is attached to a horizontal bar. This horizontal bar has guides for two parallel lift chains (one on each side of the lift tube); one end of each chain is attached to the lift carriage, the other end of each chain is attached to the lift truck body. As the lift tube piston extends itself upward, the chains draw the lift truck carriage upward. It is important to note that each link in the chain carries a load as well as the forks and the hydraulic system. The cargo rests on the forks attached to the lift carriage. Lift truck forks are usually solid steel, usually made from high strength ferritic steel.

The hydraulic system will have a fluid reservoir to provide fluid as hydraulic pistons extend (Bowman, 1972). The reservoir also allows the hydraulic oil to reject heat to the ambient environment. In normal operations, the hydraulic fluid temperature is not very high, perhaps on the order of $38^{\circ} \mathrm{C}\left(100^{\circ} \mathrm{F}\right)$ (Stewart, 1984; HPP, 1975). When hydraulic oil temperatures remain low, the lifetime of the oil is high (over 20,000 hour lifetime) (HPP, 1975). The internal combustion engine temperatures for lift trucks should be similar to those of an automobile - highest at the engine's cylinder walls (perhaps $370^{\circ} \mathrm{C}\left[700^{\circ} \mathrm{F}\right]$ in steady state) and decreasing to about 175 to $205^{\circ} \mathrm{C}\left(350\right.$ to $\left.400^{\circ} \mathrm{F}\right)$ at the outer surfaces of the engine. Data from Cole (1992) also sets some typical operating temperatures for various systems in automobiles: engine oil temperature $93^{\circ} \mathrm{C}\left(200^{\circ} \mathrm{F}\right)$, power steering fluid temperature $60^{\circ} \mathrm{C}\left(140^{\circ} \mathrm{F}\right)$, muffler exterior temperature $107^{\circ} \mathrm{C}\left(225^{\circ} \mathrm{F}\right)$, exhaust gas from the tail pipe $54^{\circ} \mathrm{C}\left(130^{\circ} \mathrm{F}\right)$. Exhaust system piping surface temperatures can be in the 90 to $260^{\circ} \mathrm{C}\left(200\right.$ to $\left.500^{\circ} \mathrm{F}\right)$ range with the high temperature at the catalytic converter (Cole, 1992). Cole (1992) also points out that when a vehicle is stopped and its engine turned off, the loss of air flow and loss of forced heat transfer from the engine coolant allows the engine temperatures to rise, perhaps up to an additional $30^{\circ} \mathrm{C}\left(85^{\circ} \mathrm{F}\right)$ above normal operating temperatures. This temperature rise can be enough to heat oil or fuel vapors above their ignition points. Cole, in discussing lift truck hydraulic oils, noted that their ignition temperature is generally about $260^{\circ} \mathrm{C}\left(500^{\circ} \mathrm{F}\right)$.

Brake systems for lift trucks are similar to automobiles, either disc brakes or drum brakes. Disc brakes have two brake shoes that are hydraulically assisted to press on each side of a rotor that is fixed to the wheel. Drum brakes have curved brake lining pads that are hydraulically assisted to press outwards onto the circumference of the drum, which is fixed to the wheel. The brake surfaces heat up from the friction of braking, perhaps in the $150^{\circ} \mathrm{C}$ $\left(300^{\circ} \mathrm{F}\right)$ range. Long duration stop-and-go driving can heat the brakes to higher temperatures. Some older lift trucks have manual brakes, but most newer lift trucks have 
the hydraulic power assist brakes. Unlike automobiles, usually only the lift truck drive wheels have brakes. The hydraulic system for brakes might have brake fluid pressure up to the $7 \mathrm{MPa}$ (1000 psig) range. Since the brake pads wear with use, there is a concern that dust from the brake pads can become airborne and inhalable. Lift truck stopping distances are usually longer than expected. An average lift truck, fully loaded and traveling at $1.6 \mathrm{kph}(1 \mathrm{mph})$ will require 0.39 meters (1.3 feet) to panic stop (Lovested, 1977). The same lift truck traveling at $16 \mathrm{kph}(10 \mathrm{mph})$ will require 6.6 meters ( $22 \mathrm{feet})$ to panic stop.

Lift trucks can make very short radius turns to accommodate close quarters such as warehouse aisles. Lift trucks are steered by the rear wheels. Directional forward steer is like an automobile (turning the steering wheel clockwise results in the vehicle turning to the right). Lift trucks can also be directional reverse steer (turning the steering wheel clockwise causes the vehicle to turn to the right when the operator is facing away from the load). Generally, only stand up rider trucks are reverse directional steer. Power steering is now standard equipment for most modern lift trucks. Power steering system hydraulic pressures are in the 7 to $21 \mathrm{MPa}$ (1000 to $3000 \mathrm{psig}$ ) range, with flows under 40 liters/minute (about $10 \mathrm{gpm}$ ). Operating temperatures are on the order of 60 to $70{ }^{\circ} \mathrm{C}$ $\left(140-160^{\circ} \mathrm{F}\right)$ (Norman, 1996). The rear steering wheels can swing outward from the body of the lift truck during turns and maneuvering, so pedestrians must beware.

There are two main styles of rider lift trucks - the outdoor or rough terrain truck that can be found at construction sites and docks, and the indoor truck used in power plants, research facilities, warehouses, factories, and industrial buildings. The rough terrain truck is usually distinguished by its large size, the large front drive wheels (although all four wheels can be large), a reinforced roll over protection system (ROPS, a cage-looking structure) around the drivers cab, and its typical use of a powerful diesel or gasoline engine. The outdoor truck often has an enclosed cab. The indoor truck is usually smaller (often under 4 meters long) than a rough terrain unit, can have the same diameter wheels in front and back, has a light construction falling object protection system (FOPS) for the driver, and can use one of the many different types of fuels discussed above. Indoor lift trucks often use solid cushion tires rather than air-filled pneumatic tires, and these trucks generally have load capacities between 1 and 6 metric tons. Cushion tires also limit the top speed of a lift truck (D'Avello, 1974). Outdoor lift trucks have load capacities of 1 to 15 , or even as high as 40, metric tons (Kulweic, 1982; Holzhauer, 1990). If pneumatic tires are used, typical air pressures are on the order of 414 to $517 \mathrm{kPa}$ (45 to $60 \mathrm{psig}$ ).

Figure 2-1 is a sketch of a typical sit down rider truck. The operator sits to drive the truck. Some lift truck models have a safety switch built in to the seat of the truck to insure that the driver is seated before the lift truck engine will start. This precaution is made since there have been injuries when drivers would disembark to see better while steering a load into a location with close tolerances. In this awkward position, sometimes the driver would run over his own toes by the need to remain very close to the vehicle to reach the controls from the ground instead of from the vehicle seat.

Other suggested safety features are rotating lights mounted high on the lift truck to call attention to the lift truck as it travels about its work path, audible back up warning signals, 


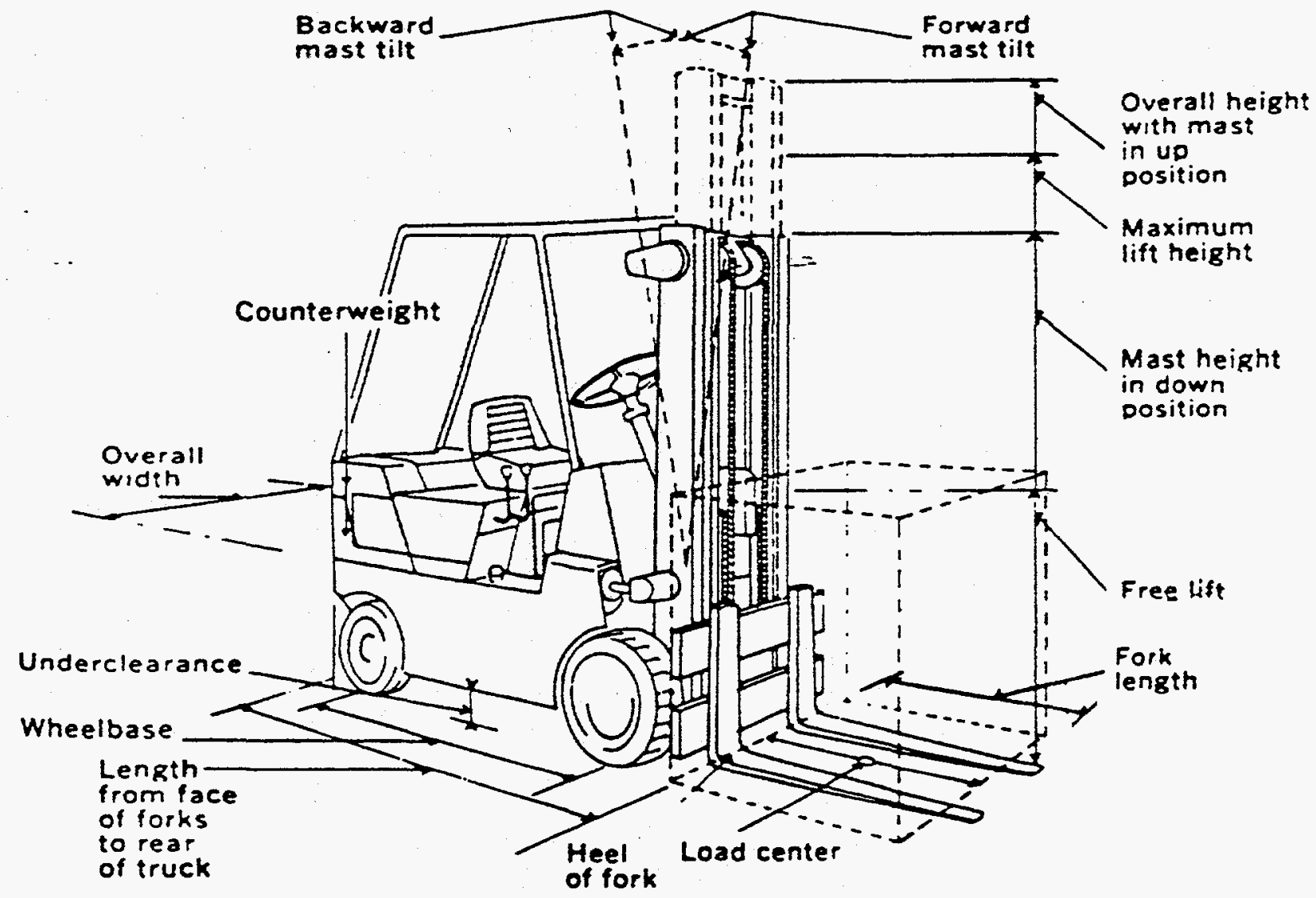

Figure 2-1. A sketch of a typical sit down rider lift truck (from Bowman, 1972). 
a built-in hydraulic one-way flow regulating valve (HPP, 1975) to slowly release hydraulic pressure so that if there is a fault with the fork hydraulic system (i.e., a burst pipe or breached hose between the lift cylinder and the control valve) the load is slowly lowered to the ground rather than quickly set down (i.e., avoids setting a load on the ground 'with authority'), a horn similar to an automobile horn to signal pedestrians and other lift truck drivers, turn signals, headlights for low light areas, fail-safe brakes, and wrap-around seats or other operator restraint systems (NSC, 1992), and safety provisions that come with the type of fuel used for the lift truck.

\section{Chapter 2 References}

Bowman, 1972. D. Bowman, Lift Trucks, A Practical Guide for Buyers and Users, Cahners Books, Boston, Massachusetts, ISBN 0-8436-1007-7, 1972.

Cole, 1992. L. S. Cole, The Investigation of Motor Vehicle Fires, third edition, Cole Books, Novato, California, ISBN 0-939818-22-1, 1992, pages $133,58 \& 102$.

D'Avello, $1974 . \quad$ F. S. D'Avello, "Reference File: Lift-Truck Tires, types and applications," Plant Engineering, 28, November 14, 1974, pages 147-150. See also "Reference File: Lift-Truck Tires, maintenance guidelines," Plant Engineering, 29, January 23, 1975, pages 141143.

Hodson, 1992. W. K. Hodson, editor, Maynard's Industrial Engineering Handbook, fourth edition, McGraw-Hill, Inc., New York, ISBN 007-041086-0, 1992, Chapter 13.

Holzhauer, 1990. R. Holzhauer, "Lift Truck Specifications Chart," Plant Engineering, 44, February 8, 1990, pages 65-85.

Holzhauer, 1992. R. Holzhauer, "Evaluating Electric Lift Trucks," Plant Engineering, 46, October 8, 1992, pages 62-66.

Holzhauer, 1997. R. Holzhauer, "What's Available in Industrial Vehicles," Plant Engineering, 51, January 1997, pages 53-62.

HPP, 1975. "Hydraulic Pneumatic Power" magazine editors, Hydraulic Handbook, fifth edition, Trade and Technical Press Ltd., Surrey, England, ISBN 85461-046-4, 1975, pages 354-364, and 469-480.

Kulweic, 1982. $\quad$ R. Kulweic, "Lift Trucks, basic types, selection factors, and engineering data," Plant Engineering, 36, July 22, 1982, pages 4456. 
Lovested, $1977 . \quad$ G. E. Lovested, "Top Ten Fork-Lift Truck Accidents," National Safety News, 116, September 1977, pages 123-127.

Norman, 1996. I. A. Norman et al., Heavy-Duty Truck Systems, second edition, Delmar Publishers, Albany New York, ISBN 0-8273-6391-5, 1996, Chapter 14.

NSC, 1992.

P. M. Laing, editor, Accident Prevention Manual for Business and Industry, engineering and technology volume, 10th edition, National Safety Council, Itasca, Illinois, ISBN 0-87912-156-4, 1992, Chapter 11.

Oliver, 1989. G. R. Oliver, "Flame Protection of Diesel and Electrical Vehicles, and Equipment," Fire Safety Engineering - Proceedings of the Second International Conference on Fire Engineering and Loss Prevention in Offshore. Petrochemical and Other Hazardous Applications, D. N. Smith, editor, Gulf Publishing Company, Houston, Texas, ISBN 0-87201-019-8, 1989, pages 191-202.

Stewart, 1984. H. L. Stewart, Pneumatics and Hydraulics, Bobbs-Merrill Company, Inc., New York, ISBN 0-672-23412-2, 1984, Chapter 1 . 


\section{Safety Issues with Lift Trucks}

The lift truck is a benefit to operations in many industries, but the lift truck also has created industrial accidents. The first part of this chapter is a hazard analysis based on energy sources utilized by lift trucks. The list of energy sources began with the list given in Johnson (1980). The latter part of this chapter presents event case histories from the literature, given in tabular form. The case histories are not all inclusive of every forklift event - time and resources would not permit such a complete treatment - but these events are indicative of recurring problems with lift trucks.

Hazards Analysis. The first step in the hazards analysis for a sit down rider lift truck is to identify all the energy sources that the lift truck uses or controls. Based on the description of the lift truck given in the previous chapter, applicable energy sources are:

\begin{tabular}{|l|l|}
\hline Energy type & Hazards associated with energy type \\
\hline kinetic energy of vehicle & $\begin{array}{l}\text { - collision with person, cargo, building elements } \\
\text { - collision with other equipment } \\
\text { - fork impact onto person, cargo, building or equipment } \\
\text { - truck can push an object } \\
\text { - tire can throw or sling small debris the truck runs over } \\
\text { - truck can run over object }\end{array}$ \\
\hline $\begin{array}{l}\text { kinetic energy of load } \\
\text { motion }\end{array}$ & $\begin{array}{l}\text { - impact of load on person, other cargo, building elements } \\
\text { - impact of load on shelf, ceiling beams, or equipment }\end{array}$ \\
\hline mechanical energy of mast & $\begin{array}{l}\text { - pinch point } \\
\text { as it is tilted } \\
\text { - load can be unstable if tilted in wrong direction }\end{array}$ \\
\hline mechanical energy of shafts & $\begin{array}{l}\text { - possible injury if worker is pulled to shaft via clothing } \\
\text { or hair, etc. } \\
\text { - shaft rotation could throw debris after a material failure, } \\
\text { such as a hydraulic pump catastrophic failure }\end{array}$ \\
\hline $\begin{array}{l}\text { hydraulic oil pressure } \\
\text { energy }\end{array}$ & $\begin{array}{l}\text { - high pressure can lead to leaks; leaks can present a } \\
\text { possible fire hazard } \\
\text { - health issue, pressure can inject oil through skin }\end{array}$ \\
\hline hydraulic oil thermal energy \\
energy
\end{tabular}




\begin{tabular}{|c|c|}
\hline $\begin{array}{l}\text { brake system pathogenic } \\
\text { dust }\end{array}$ & $\begin{array}{l}\text { - dust ablation from brakes can create inhalable aerosol } \\
\text { particles that are hazardous to health }\end{array}$ \\
\hline acoustic energy from engine & $\begin{array}{l}\text { - vehicle operation noise can preclude operator from } \\
\text { hearing important sounds (instructions or warnings, } \\
\text { pager, fire alarm, etc.) over } 85 \mathrm{~dB}-\mathrm{A} \text { can be harmful to } \\
\text { the operator's hearing }\end{array}$ \\
\hline $\begin{array}{l}\text { vibration energy from } \\
\text { engine }\end{array}$ & $\begin{array}{l}\text { - lift truck operator can be fatigued, and vibration can lead } \\
\text { to mechanical component failure }\end{array}$ \\
\hline potential energy of lift truck & $\begin{array}{l}\text { - lift truck can fall off of a ledge or a dock plate } \\
\text { - fall through the floor of a road truck trailer (if faulty) } \\
\text { - fall through weak covers of trenches or openings in floor } \\
\text { - if road truck trailer is not secured, lift truck can cause it } \\
\text { to rock or pivot, which could lead to tipping the lift truck } \\
\text { - lift truck can topple over on a grade or when making a } \\
\text { sharp turn } \\
\text { - lift truck can be pulled over when being towed by another } \\
\text { vehicle } \\
\text { - abrupt stop can cause load to travel on, leaving the forks }\end{array}$ \\
\hline potential energy of load & $\begin{array}{l}\text { - imbalance on forks can lead to dropping load } \\
\text { - imbalance on forks can lead to toppling lift truck } \\
\text { - falling object protection system or backrest extension } \\
\text { protects operator from load tipping back onto the lift truck } \\
\text { - lifting a load beyond truck capacity can over-balance the } \\
\text { truck or damage the fork tines }\end{array}$ \\
\hline $\begin{array}{l}\text { electrical energy of lift truck } \\
\text { battery }\end{array}$ & $\begin{array}{l}\text { - electrical arc, sparks } \\
\text { - batteries contain corrosive acid }\end{array}$ \\
\hline $\begin{array}{l}\text { combustible substances used } \\
\text { on lift trucks }\end{array}$ & $\begin{array}{l}\text { - fuel (gasoline, diesel fuel, propane, natural gas) } \\
\text { - grease for lubrication } \\
\text { - engine oil, if needed for the engine type } \\
\text { - hydraulic fluid (usually flame resistant, but can still burn) } \\
\text { - power steering fluid } \\
\text { - automatic transmission fluid } \\
\text { As stated above, all of these fuels and oils could lead to a } \\
\text { fire, which can burn and lead to inhalation of toxic smoke } \\
\text { products and to property damage }\end{array}$ \\
\hline
\end{tabular}




\begin{tabular}{|c|c|}
\hline $\begin{array}{l}\text { pathogenic issues with lift } \\
\text { truck fuels and lubricants }\end{array}$ & $\begin{array}{l}\text { - fuel (gasoline, diesel fuel, propane, natural gas) } \\
\text { - grease for lubrication } \\
\text { - engine oil, if needed for the engine type } \\
\text { - hydraulic fluid (usually flame resistant, but can still burn) } \\
\text { - power steering fluid } \\
\text { - transmission fluid } \\
\text { - engine coolant (i.e., ethylene glycol) } \\
\text { These substances may pose health threats for workers } \\
\text { exposed to them on a routine basis. This may be more of } \\
\text { an issue for maintainers and refuelers than lift truck } \\
\text { operators. }\end{array}$ \\
\hline engine thermal energy & $\begin{array}{l}\text { - burns } \\
\text { - possible heat ignition of some combustible substances } \\
\text { - may lead to operator heat exhaustion }\end{array}$ \\
\hline $\begin{array}{l}\text { engine exhaust thermal } \\
\text { energy }\end{array}$ & $\begin{array}{l}\text { - burns from hot parts (muffler and manifold piping) } \\
\text { - possible ignition of combustible substances } \\
\text { - exhaust is an inhalation health hazard } \\
\text { - possible oxygen deficiency if lift truck is used in confined } \\
\text { area }\end{array}$ \\
\hline air pressure energy in tires & $\begin{array}{l}\text { - pressure can propel debris, debris could cause impact } \\
\text { injury }\end{array}$ \\
\hline $\begin{array}{l}\text { human effort to operate lift } \\
\text { truck }\end{array}$ & $\begin{array}{l}\text { - repetitive motion injuries } \\
\text { - driver fatigue } \\
\text { - slips, trips, or falls when driver is embarking or } \\
\text { disembarking lift truck } \\
\text { - slips, trips or falls of passenger riding on truck body or } \\
\text { on forks } \\
\text { - slips, trips or falls when worker is climbing on top of lift } \\
\text { truck falling object protection system to access shelves }\end{array}$ \\
\hline $\begin{array}{l}\text { human effort working with } \\
\text { lift truck }\end{array}$ & $\begin{array}{l}\text { - improper use of lift truck as an elevator } \\
\text { - improper use of a lift truck as a taxi cab } \\
\text { - people standing too close to path of truck can be struck } \\
\text { or have their feet run over }\end{array}$ \\
\hline
\end{tabular}

Operating Experiences. The next step in hazards analysis is to review past events. Various publications were reviewed to better understand lift truck accidents. The following list gives brief narratives and reference citations for lift truck events. 


\begin{tabular}{|l|l|}
\hline Reference & Brief Narrative of Lift Truck-related Event or Accident \\
\hline NFPA, 1972 & $\begin{array}{l}\text { A forklift on the aircraft carrier USS Constellation pushed a bin } \\
\text { into a metal plate, the plate in turn ruptured a fuel oil pipe. Ignition } \\
\text { from a source other than the lift truck started a fuel oil fire, killing } \\
50 \text { crewmen and destroying the ship for a \$48 million dollar loss. }\end{array}$ \\
\hline Booth, 1979 & $\begin{array}{l}\text { Two events are discussed: a collision between two lift trucks } \\
\text { when two drivers disputed the right-of-way; and a truck with } \\
\text { defective brakes that gained speed down a ramp and hit a column, } \\
\text { causing the driver such injury that his leg had to be amputated. }\end{array}$ \\
\hline $\begin{array}{l}\text { Williams and } \\
\text { Priestly, 1980 }\end{array}$ & $\begin{array}{l}\text { Driver caught under a lift truck when it overturned } \\
\text { Worker caught between a lift truck and a building wall } \\
\text { Lift truck driver was loading steel bars of unequal length onto } \\
\text { a road truck. The lift truck driver saw one steel bar protruding } \\
\text { and used the lift truck to push the bar forwards. The bar entered } \\
\text { the road truck cab, penetrating the road truck driver's calf. }\end{array}$ \\
\hline Marryatt, 1988 & $\begin{array}{l}\text { Over 100 years of data, only 14 forklift truck fires have been } \\
\text { recorded in Australia and New Zealand. The Australians believe } \\
\text { the low fire frequency has been partly due to the widespread } \\
\text { conversion from gasoline to liquefied petroleum fuels. }\end{array}$ \\
\hline Kletz, 1988 & $\begin{array}{l}\text { Full 40-gallon drums were being loaded onto a road trailer by a lift } \\
\text { truck. The lift truck placed two drums at a time on their sides } \\
\text { (onto the rolling hoops) and then tipped them upright using the } \\
\text { forks. One drum tipped too far and fell over, onto the foot of the } \\
\text { road truck driver, who was standing on the road trailer to adjust } \\
\text { the position of the drums. }\end{array}$ \\
\hline
\end{tabular}




\begin{tabular}{|c|c|}
\hline O'Mara, 1989 & $\begin{array}{l}6 \text { fatal accidents involving lift trucks between } 1980-1988 \text { : } \\
\text { - The operator of a power lift stacker was lifting pallets from the } \\
\text { mezzanine floor to the ground floor when the forks became caught } \\
\text { in a channel on the front of the mezzanine. The lift truck wheels } \\
\text { lifted off the floor. When the forks unstuck, the machine fell to } \\
\text { the floor and overbalanced, the operator was crushed. } \\
\text { - The operator was moving food into a freezer room. He was } \\
\text { found lying on the floor of the freezer room next to the idling lift } \\
\text { truck. The lift truck carburetor was emitting high levels of carbon } \\
\text { monoxide. The autopsy showed that the operator had been } \\
\text { overcome with carbon monoxide poisoning. } \\
\text { - A worker was crushed by rolls of paper tissue. A lift truck } \\
\text { bumped a stack of } 14 \text { rolls of paper, which dominoed into another } \\
\text { stack, and this effect continued on and fell on another worker. } \\
\text { - A lift truck driver picked up a lead/steel load during a dismantle } \\
\text { operation. The load caught on the floor and applied an overturning } \\
\text { moment on the lift truck. The truck overturned, trapping the driver } \\
\text { between the overhead protection guard and the ground. } \\
\text { - While driving a lift truck, the operator did not notice his } \\
\text { clearance to the overhead. The extended mast struck an overhead } \\
\text { steel beam, causing the truck to overturn. The lift truck tipped } \\
\text { backward, crushing the driver. } \\
\text { - The lift truck driver's son was helping his father by driving the } \\
\text { lift truck to carry out job duties. The son overbalanced the lift } \\
\text { truck, it rolled onto the son. The son was not trained and was too } \\
\text { young to be driving a lift truck. }\end{array}$ \\
\hline & $\begin{array}{l}\text { There were } 861 \text { forklift related accidents recorded in } 1980-1986 \text { in } \\
\text { Australia. Five resulted in permanent disability, } 91 \text { were partial } \\
\text { impairment, and } 762 \text { were temporary disability cases. There were } \\
3 \text { fatalities in that time period. }\end{array}$ \\
\hline Cole, 1992 & $\begin{array}{l}\text { A lift truck caught fire, witnesses saw smoke and flames coming } \\
\text { from the rear of the engine compartment. Investigation revealed } \\
\text { that the positive cable to the vehicle battery (i.e., the starter motor } \\
\text { battery) had been vibrating and rubbing against the metal braided } \\
\text { cover of a hydraulic line. The battery cable insulation had worn } \\
\text { away and the cable shorted to the metal of the hydraulic line. With } \\
\text { an arc temperature over } 1037^{\circ} \mathrm{C}\left(1900^{\circ} \mathrm{F}\right) \text {, the hydraulic line failed } \\
\text { and allowed oil to escape. The arc ignited the escaping oil. An } \\
\text { intense fire followed. Cole stated that construction vehicle fires } \\
\text { are often electrical, followed by fuel leaks, friction heat buildup, } \\
\text { and overheating from hard usage. }\end{array}$ \\
\hline DOE ESH, 1992 & $\begin{array}{l}\text { A pedestrian was killed in May } 1991 \text { when struck by a lift truck, } \\
\text { and a lift truck operator was severely injured in March } 1992 \text { when } \\
\text { he backed his lift truck off the end of a loading dock. }\end{array}$ \\
\hline
\end{tabular}




\begin{tabular}{|l|l|}
\hline OSO, 1993 & $\begin{array}{l}\text { A lift truck carrying a large load was being driven forward so that } \\
\text { the driver could monitor the load and ensure that it was not tipping } \\
\text { from the lift truck. A platform lift driver was standing in the aisle } \\
\text { way performing another task when the lift truck struck the } \\
\text { platform lift and the platform lift driver. She suffered a broken leg } \\
\text { as the lift truck tine struck her. The lift truck stopped before any } \\
\text { more serious injury could occur. }\end{array}$ \\
\hline $\begin{array}{l}\text { Larsson and } \\
\text { Rechnitzer, 1994 }\end{array}$ & $\begin{array}{l}\text { Fifteen forklift related fatality events in Australia between 1987- } \\
1990: \\
1 \text { child fell from a forklift as it reversed direction at a party held } \\
\text { in the workplace } \\
5 \text { drivers were run over by their own vehicle as they worked to } \\
\text { adjust cargo or other equipment } \\
1 \text { driver was crushed by the forklift load } \\
2 \text { workers suffered fatal falls from the forklift tines } \\
4 \text { workers were killed when struck by the lift truck } \\
2 \text { workers were crushed to death by shifting lift truck loads }\end{array}$ \\
\hline OSHA, 1996 & $\begin{array}{l}\text { A worker went underneath a load on the lift truck to make a } \\
\text { measurement of the load. The load handling modification } \\
\text { disengaged from the lift truck carriage and dropped the } 4550 \mathrm{~kg} \\
\text { (10,000 pound) load onto the worker, killing him. }\end{array}$ \\
\hline
\end{tabular}

The US Department of Energy maintains a data base of injuries. This data base is called the Safety Performance Measurement System. A search over the time from the beginning of 1991 to the end of 1996 showed many small injury events with lift trucks. Some of the one-line descriptions of these events are given in the following list. The one-line descriptions are generally straightforward and provide the reader with an overview of the event and the injury that occurred. Interested readers can access the data base and use the facility number and case number to retrieve the full text discussion of the event. 


\begin{tabular}{|l|l|l|}
\hline $\begin{array}{l}\text { SPMS case } \\
\text { number }\end{array}$ & Event date & One-line description of event \\
\hline $\begin{array}{l}\text { facility } \\
0515002\end{array}$ & & \\
\hline 92010 & $03 / 10 / 92$ & $\begin{array}{l}\text { Strained lower back while climbing over arm rest to } \\
\text { dismount forklift }\end{array}$ \\
\hline 92066 & $09 / 09 / 92$ & $\begin{array}{l}\text { Hit bump in road while operating forklift and } \\
\text { contused lower back }\end{array}$ \\
\hline 92501 & $02 / 06 / 92$ & $\begin{array}{l}\text { 1-ton weight fell during transport and damaged } \\
\text { forklift }\end{array}$ \\
\hline 92510 & $12 / 09 / 92$ & Rear wheel of forklift broke off axle during transit \\
\hline 93065 & $06 / 08 / 93$ & $\begin{array}{l}\text { Fractured finger between tines and safety latch } \\
\text { while using forklift }\end{array}$ \\
\hline 93099 & $08 / 14 / 93$ & $\begin{array}{l}\text { Strained back while twisting to put shelves onto } \\
\text { tines of forklift }\end{array}$ \\
\hline 94062 & $05 / 17 / 94$ & $\begin{array}{l}\text { Foreign body entered eye while operating forklift, } \\
\text { causing irritation }\end{array}$ \\
\hline 94143 & $11 / 03 / 94$ & $\begin{array}{l}\text { Contused/abraded leg while attempting to remount } \\
\text { moving forklift }\end{array}$ \\
\hline 94149 & $12 / 07 / 94$ & $\begin{array}{l}\text { Wheel ran into foot while pushing equipment, } \\
\text { causing contusion to toe }\end{array}$ \\
\hline 95025 & $03 / 11 / 95$ & $\begin{array}{l}\text { Caught hand between pallets while moving, } \\
\text { causing laceration }\end{array}$ \\
\hline 95044 & $04 / 08 / 95$ & $\begin{array}{l}\text { Wind blew shelf off forklift and shelf struck head, } \\
\text { causing multiple strains }\end{array}$ \\
\hline 95070 & $06 / 20 / 95$ & $\begin{array}{l}\text { Caught hand between forklift and box, caused } \\
\text { amputation of fingertip }\end{array}$ \\
\hline 95098 & $07 / 24 / 95$ & $\begin{array}{l}\text { Developed plantar fascitis from continually getting } \\
\text { on and off a forklift }\end{array}$ \\
\hline 95161 & $12 / 11 / 95$ & $\begin{array}{l}\text { Tines dropped and pinched finger between stand } \\
\text { and tines, fracture resulted }\end{array}$ \\
\hline 96054 & $05 / 07 / 96$ & $\begin{array}{l}\text { Lacerated finger on tooling while positioning } \\
\text { tooling on forklift tines }\end{array}$ \\
\hline $\begin{array}{l}\text { facility } \\
0531002\end{array}$ & & Load on forklift became unstable and fell over \\
\hline 95047 & $03 / 20 / 95$ & $\begin{array}{l}\text { Cutter-grinder fell off forklift and fractured foot } \\
\text { while being moved }\end{array}$ \\
\hline 95089 & $\begin{array}{l}\text { Stepped on bolt in walkway while exiting fork } \\
\text { truck and strained knee }\end{array}$ \\
\hline 95108 & $07 / 11 / 95$ & \\
\hline $95 / 09 / 95$ & &
\end{tabular}




\begin{tabular}{|c|c|c|}
\hline $\begin{array}{l}\text { SPMS case } \\
\text { number }\end{array}$ & Event date & One-line description of event \\
\hline \multicolumn{3}{|l|}{$\begin{array}{l}\text { facility } \\
0544003\end{array}$} \\
\hline 92027 & $01 / 02 / 92$ & $\begin{array}{l}\text { Plywood struck and contused foot when ejected } \\
\text { from under tire of forklift }\end{array}$ \\
\hline 92240 & $07 / 07 / 92$ & $\begin{array}{l}\text { Forklift fixture fell during maintenance work, } \\
\text { causing unspecified injury }\end{array}$ \\
\hline 92322 & $09 / 03 / 92$ & $\begin{array}{l}\text { Hydraulic line on forklift truck broke and chemical } \\
\text { abraded both eyes }\end{array}$ \\
\hline 94144 & $06 / 16 / 94$ & $\begin{array}{l}\text { Fencing being lowered onto forklift brushed } \\
\text { against leg, causing abrasion }\end{array}$ \\
\hline 94151 & $07 / 01 / 94$ & $\begin{array}{l}\text { Finger was injured by rotating steering wheel knob } \\
\text { when forklift struck a hole }\end{array}$ \\
\hline 94276 & 10/03/94 & $\begin{array}{l}\text { Slipped while dismounting forklift, causing bruise } \\
\text { to abdomen }\end{array}$ \\
\hline 94299 & $12 / 15 / 94$ & $\begin{array}{l}\text { Pickup truck was struck by forklift tongs while } \\
\text { unloading cylinders from pickup }\end{array}$ \\
\hline 95304 & $06 / 21 / 95$ & $\begin{array}{l}\text { Strained arm when brake was applied and forklift } \\
\text { brake lever stuck }\end{array}$ \\
\hline 95524 & $11 / 22 / 95$ & $\begin{array}{l}\text { Forklift rolled into depression, trapping employee } \\
\text { under forklift rollover cage }\end{array}$ \\
\hline \multicolumn{3}{|l|}{$\begin{array}{l}\text { facility } \\
0544904\end{array}$} \\
\hline 92124 & $06 / 29 / 92$ & $\begin{array}{l}\text { Strained shoulder while lifting hood of forklift to } \\
\text { perform maintenance }\end{array}$ \\
\hline 92171 & $09 / 02 / 92$ & $\begin{array}{l}\text { Hand was caught in pinch point while operating } \\
\text { forklift, causing avulsion }\end{array}$ \\
\hline 93032 & $02 / 26 / 93$ & Lacerated knuckle on forklift while being repaired \\
\hline 93216 & $11 / 23 / 93$ & $\begin{array}{l}\text { Strained shoulder while lifting tire to be replaced on } \\
20 \text {-ton forklift }\end{array}$ \\
\hline 95059 & $05 / 09 / 95$ & $\begin{array}{l}\text { Spreader slipped from forks of forklift and struck } \\
\text { hand, causing laceration }\end{array}$ \\
\hline 960404 & $03 / 26 / 96$ & Struck forehead on forklift, causing laceration \\
\hline 96091 & $06 / 28 / 96$ & $\begin{array}{l}\text { Hood of forklift dropped down on arm, resulting in } \\
\text { forearm contusion }\end{array}$ \\
\hline \multicolumn{3}{|l|}{$\begin{array}{l}\text { facility } \\
0553002 \\
\end{array}$} \\
\hline 96013 & $08 / 26 / 96$ & $\begin{array}{l}\text { Exited forklift and stepped } 1.5 \text { feet from step to } \\
\text { ground, lower leg pain }\end{array}$ \\
\hline \multicolumn{3}{|l|}{$\begin{array}{l}\text { facility } \\
0577004\end{array}$} \\
\hline 92024 & $11 / 19 / 92$ & $\begin{array}{l}\text { Forklift lost power on ramp during operation and } \\
\text { rolled into aircraft }\end{array}$ \\
\hline
\end{tabular}




\begin{tabular}{|l|l|l|}
\hline $\begin{array}{l}\text { SPMS case } \\
\text { number }\end{array}$ & Event date & One-line description of event \\
\hline $\begin{array}{l}\text { facility } \\
0578003\end{array}$ & $02 / 12 / 92$ & $\begin{array}{l}\text { Sheet metal fell and fractured foot while being } \\
\text { unloaded from forklift }\end{array}$ \\
\hline 92027 & $04 / 01 / 92$ & $\begin{array}{l}\text { Stumbled on pallet and reached out, jamming } \\
\text { thumb on forklift door }\end{array}$ \\
\hline 92050 & $05 / 14 / 92$ & $\begin{array}{l}\text { Forearm was burned on wand while using steam } \\
\text { cleaner to clean forklift }\end{array}$ \\
\hline 92103 & $04 / 02 / 92$ & $\begin{array}{l}\text { Aggravated knee surgery by repetitively climbing } \\
\text { stairs and mounting forklift }\end{array}$ \\
\hline 92113 & $\begin{array}{l}\text { Tripped over times of forklift and fell, abrading } \\
\text { knee }\end{array}$ \\
\hline 93092 & $04 / 22 / 93$ & $\begin{array}{l}\text { Strained shoulder and calf while stepping down } \\
\text { from forklift to pallet }\end{array}$ \\
\hline 93096 & $02 / 08 / 93$ & $\begin{array}{l}\text { Overexerted groin lifting 100 pound tire from } \\
\text { forklift, causing hernia }\end{array}$ \\
\hline 93158 & $08 / 27 / 93$ & $\begin{array}{l}\text { Hand was contused by falling forklift hood when } \\
\text { screwdriver arced against battery }\end{array}$ \\
\hline 94083 & $04 / 21 / 94$ & $\begin{array}{l}\text { Hip/buttocks was struck/injured by palletized } \\
\text { material on moving forklift }\end{array}$ \\
\hline 94122 & $04 / 11 / 94$ & $\begin{array}{l}\text { Drove forklift in dip in road, which resulted in } \\
\text { lower back spasm }\end{array}$ \\
\hline 95118 & $07 / 10 / 95$ & $\begin{array}{l}\text { Backed out of forklift, slipped on step which } \\
\text { resulted in hip pain }\end{array}$ \\
\hline 95121 & $07 / 25 / 95$ & $\begin{array}{l}\text { Pinned side on forklift/rack while backing and } \\
\text { turning rack to pull material }\end{array}$ \\
\hline 96023 & $03 / 19 / 96$ & $\begin{array}{l}\text { Tines caught on bump while pulling pallet, caused } \\
\text { shoulder strain }\end{array}$ \\
\hline 96033 & $03 / 26 / 96$ & $\begin{array}{l}\text { Seat struck shoulder/neck after lifting seating } \\
\text { compartment, resulted in contusions }\end{array}$ \\
\hline 96069 & $02 / 27 / 96$ & $\begin{array}{l}\text { Struck head on forklift cab while entering, caused } \\
\text { neck sprain }\end{array}$ \\
\hline 96133 & $09 / 09 / 96$ & $\begin{array}{l}\text { Fork slid from carriage of forklift and fell, } \\
\text { contusing foot }\end{array}$ \\
\hline 93126 & $10 / 28 / 93$ & $\begin{array}{l}\text { Forklift tine fell when caught on rail track and } \\
\text { struck/fractured finger }\end{array}$ \\
\hline 94072 & $08 / 11 / 94$ & $\begin{array}{l}\text { Strained elbow and forearm while adjusting metal } \\
\text { beams on forklift tines }\end{array}$ \\
\hline 96011 & $05 / 10 / 96$ & \\
\hline
\end{tabular}




\begin{tabular}{|c|c|c|}
\hline $\begin{array}{l}\text { SPMS case } \\
\text { number }\end{array}$ & Event date & One-line description of event \\
\hline \multicolumn{3}{|l|}{\begin{tabular}{l|l} 
facility \\
1001003 \\
\end{tabular}} \\
\hline 92059 & $01 / 27 / 92$ & $\begin{array}{l}\text { Piece of wood became airborne and contused leg } \\
\text { when run over by forklift }\end{array}$ \\
\hline 92111 & $08 / 03 / 92$ & $\begin{array}{l}\text { Slipped while positioning cabinet onto forklift and } \\
\text { lacerated head on frame }\end{array}$ \\
\hline \multicolumn{3}{|l|}{$\begin{array}{l}\text { facility } \\
1002503\end{array}$} \\
\hline 92041 & $06 / 05 / 92$ & $\begin{array}{l}\text { Forklift rolled onto foot when parking brake failed, } \\
\text { resulting in fracture }\end{array}$ \\
\hline 93097 & $10 / 25 / 93$ & $\begin{array}{l}\text { Strained neck when fork truck dropped into } \\
\text { pavement while operating }\end{array}$ \\
\hline 96061 & $08 / 05 / 96$ & $\begin{array}{l}\text { Incurred multiple injuries from daily work of } \\
\text { driving and lifting packages }\end{array}$ \\
\hline \multicolumn{3}{|l|}{$\begin{array}{l}\text { facility } \\
1002506\end{array}$} \\
\hline 940003 & $11 / 30 / 94$ & $\begin{array}{l}\text { While unloading waterproofing rolls, fell and } \\
\text { struck head on forklift tire }\end{array}$ \\
\hline \multicolumn{3}{|l|}{$\begin{array}{l}\text { facility } \\
1005003\end{array}$} \\
\hline 92065 & $11 / 12 / 92$ & $\begin{array}{l}\text { Caught foot in tines of forklift and fell, causing } \\
\text { multiple injuries }\end{array}$ \\
\hline 96010 & $04 / 09 / 96$ & Lid cover slipped off forklift and crushed fingers \\
\hline \multicolumn{3}{|l|}{$\begin{array}{l}\text { facility } \\
2806006 \\
\end{array}$} \\
\hline 93008 & 09/14/93 & $\begin{array}{l}\text { Stepped onto dirt clod stepping off of forklift and } \\
\text { twisted ankle }\end{array}$ \\
\hline \multicolumn{3}{|l|}{$\begin{array}{l}\text { facility } \\
3005003\end{array}$} \\
\hline 92025 & $04 / 30 / 92$ & $\begin{array}{l}\text { Eye was struck by particulate matter while moving } \\
\text { drums with forklift }\end{array}$ \\
\hline 92032 & $06 / 11 / 92$ & $\begin{array}{l}\text { Forklift struck edge of ramp, causing steering } \\
\text { wheel knob to fracture hand }\end{array}$ \\
\hline 93028 & 03/08/93 & $\begin{array}{l}\text { Strained shoulder lifting forklift cab to gain access } \\
\text { to forklift battery }\end{array}$ \\
\hline 93067 & $01 / 14 / 93$ & $\begin{array}{l}\text { Slipped and fell, injured tailbone against forklift } \\
\text { tires }\end{array}$ \\
\hline 94030 & $03 / 14 / 94$ & $\begin{array}{l}\text { Pulled muscle in rib cage while positioning pallet } \\
\text { jack on forklift tines }\end{array}$ \\
\hline 94131 & $09 / 22 / 94$ & Lacerated lower leg while exiting cab of forklift \\
\hline
\end{tabular}




\begin{tabular}{|c|c|c|}
\hline $\begin{array}{l}\text { SPMS case } \\
\text { number }\end{array}$ & Event date & One-line description of event \\
\hline \multicolumn{3}{|l|}{$\begin{array}{l}\text { facility } \\
3501204\end{array}$} \\
\hline 93002 & $01 / 25 / 92$ & $\begin{array}{l}\text { X-ray machine tipped over into wall while being } \\
\text { moved onto forklift tines }\end{array}$ \\
\hline 94034 & $09 / 09 / 94$ & $\begin{array}{l}\text { Caught and lacerated finger while reinstalling tines } \\
\text { on forklift }\end{array}$ \\
\hline 95026 & $10 / 10 / 95$ & $\begin{array}{l}\text { Strained wrist while pushing self away from pod } \\
\text { that was falling from forklift }\end{array}$ \\
\hline 96012 & $06 / 24 / 96$ & Strained neck while stooping to go under forklift \\
\hline \multicolumn{3}{|l|}{$\begin{array}{l}\text { facility } \\
3501405 \\
\end{array}$} \\
\hline 92010 & $01 / 10 / 92$ & $\begin{array}{l}\text { Injured head/back when struck by cross member } \\
\text { being pulled by forklift }\end{array}$ \\
\hline 92025 & $02 / 06 / 92$ & $\begin{array}{l}\text { Struck bump while operating forklift and bounced } \\
\text { up, injuring neck and back }\end{array}$ \\
\hline 92040 & $02 / 25 / 92$ & $\begin{array}{l}\text { Particle entered eye during operations of forklift, } \\
\text { causing conjunctivitis }\end{array}$ \\
\hline 92176 & $04 / 14 / 92$ & $\begin{array}{l}\text { Forklift operator backed into manlift while moving } \\
\text { bin, damaged manlift }\end{array}$ \\
\hline 92178 & $08 / 18 / 92$ & Arm was bitten by insect while driving forklift \\
\hline 92183 & $07 / 28 / 92$ & $\begin{array}{l}\text { Forklift carrying } 8000 \text { pound load damaged a } \\
\text { torque wrench when forklift brakes failed }\end{array}$ \\
\hline 92228 & $10 / 19 / 92$ & $\begin{array}{l}\text { Scissor lift fell to ground and sustained damage } \\
\text { when elevated by a forklift }\end{array}$ \\
\hline 92961 & $06 / 29 / 92$ & $\begin{array}{l}\text { Forklift fell and was damaged while being } \\
\text { unloaded onto trailer }\end{array}$ \\
\hline 93031 & $03 / 01 / 93$ & $\begin{array}{l}250 \text { pound hood fell and lacerated skull during } \\
\text { replacement of forklift battery }\end{array}$ \\
\hline 93038 & $03 / 12 / 93$ & $\begin{array}{l}\text { Forklift operator lowered } 20,000 \text { pound steel I- } \\
\text { beam onto foot, causing injuries }\end{array}$ \\
\hline 93049 & $03 / 23 / 93$ & $\begin{array}{l}\text { Load on forklift shifted to one tine, breaking the } \\
\text { forklift tine }\end{array}$ \\
\hline 93081 & $05 / 24 / 93$ & $\begin{array}{l}\text { Slipped off side of forklift after being startled by } \\
\text { snake, sprained back }\end{array}$ \\
\hline 93131 & $07 / 29 / 93$ & $\begin{array}{l}\text { Leg struck against forklift tine while laying cable, } \\
\text { lacerating lower leg }\end{array}$ \\
\hline 93175 & $11 / 2 / 93$ & $\begin{array}{l}\text { Wheel/hub suddenly released and struck leg while } \\
\text { removing, causing fracture }\end{array}$ \\
\hline 94021 & $03 / 01 / 94$ & $\begin{array}{l}\text { Van struck parked forklift when van operator's foot } \\
\text { became tangled on pedal }\end{array}$ \\
\hline 94035 & $04 / 20 / 94$ & $\begin{array}{l}\text { Struck head against roll cage while exiting forklift, } \\
\text { straining neck }\end{array}$ \\
\hline
\end{tabular}




\begin{tabular}{|c|c|c|}
\hline $\begin{array}{l}\text { SPMS case } \\
\text { number }\end{array}$ & Event date & One-line description of event \\
\hline \multicolumn{3}{|l|}{$\begin{array}{l}\text { facility } \\
3501405, \\
\text { continued }\end{array}$} \\
\hline 94073 & $07 / 08 / 94$ & $\begin{array}{l}\text { Wind caught door and struck elbow while entering } \\
\text { forklift, causing contusion }\end{array}$ \\
\hline 95047 & $03 / 27 / 95$ & $\begin{array}{l}\text { Forklift tine broke off while attempting to dig } \\
\text { pedestal out of ground }\end{array}$ \\
\hline \multicolumn{3}{|l|}{$\begin{array}{l}\text { facility } \\
4002502\end{array}$} \\
\hline 92020 & $01 / 08 / 92$ & $\begin{array}{l}\text { Plexiglas enclosure on forklift was damaged due to } \\
\text { unidentified stress }\end{array}$ \\
\hline 92117 & $11 / 24 / 92$ & $\begin{array}{l}\text { Slipped and contused wrist while descending from } \\
\text { forklift }\end{array}$ \\
\hline \multicolumn{3}{|l|}{$\begin{array}{l}\text { facility } \\
4005106\end{array}$} \\
\hline 94025 & $10 / 22 / 94$ & $\begin{array}{l}\text { Injured back while removing barrel grabbers from } \\
\text { forklift }\end{array}$ \\
\hline 95022 & $11 / 16 / 95$ & $\begin{array}{l}\text { Forklift pan came down and struck foot while } \\
\text { loading base sheet, contused foot }\end{array}$ \\
\hline \multicolumn{3}{|l|}{$\begin{array}{l}\text { facility } \\
4006002\end{array}$} \\
\hline 92030 & $04 / 13 / 92$ & $\begin{array}{l}\text { Contused leg against fork of forklift while } \\
\text { unloading materials from truck }\end{array}$ \\
\hline 92039 & $04 / 01 / 92$ & $\begin{array}{l}\text { Developed bursitis in arm after tripping over tines } \\
\text { of forklift }\end{array}$ \\
\hline 92111 & $10 / 05 / 92$ & $\begin{array}{l}\text { Foot slipped off brake and forklift struck column, } \\
\text { causing whiplash injury }\end{array}$ \\
\hline 92154 & $12 / 24 / 92$ & $\begin{array}{l}\text { Strained shoulder while grabbing steering wheel to } \\
\text { enter forklift }\end{array}$ \\
\hline 93042 & $04 / 21 / 93$ & $\begin{array}{l}\text { Incurred contusions to head and leg when forklift } \\
\text { struck against column }\end{array}$ \\
\hline 93088 & $06 / 10 / 93$ & $\begin{array}{l}\text { Strained back while turning to look backward in } \\
\text { seatbelt of forklift }\end{array}$ \\
\hline 94064 & $05 / 17 / 94$ & $\begin{array}{l}\text { Injured medial compartment of knee while } \\
\text { dismounting forklift }\end{array}$ \\
\hline$\overline{94147}$ & $09 / 14 / 94$ & $\begin{array}{l}\text { Strained upper back while emptying 5-gallon fuel } \\
\text { cans into forklift trucks }\end{array}$ \\
\hline 95069 & $06 / 23 / 95$ & $\begin{array}{l}\text { Driving a forklift, felt substance in eye, diagnosed } \\
\text { as corneal abrasion }\end{array}$ \\
\hline 96111 & $09 / 16 / 96$ & $\begin{array}{l}\text { Forklift rounded corner and struck chest, causing } \\
\text { abrasions and contusions }\end{array}$ \\
\hline
\end{tabular}




\begin{tabular}{|c|c|c|}
\hline $\begin{array}{l}\text { SPMS case } \\
\text { number }\end{array}$ & Event date & One-line description of event \\
\hline \multicolumn{3}{|l|}{$\begin{array}{l}\text { facility } \\
4006004\end{array}$} \\
\hline 92006 & $09 / 14 / 92$ & $\begin{array}{l}\text { Incurred neck/shoulder strain when forklift backed } \\
\text { into another forklift }\end{array}$ \\
\hline \multicolumn{3}{|l|}{$\begin{array}{l}\text { facility } \\
4006503\end{array}$} \\
\hline 93034 & $03 / 31 / 93$ & $\begin{array}{l}\text { Monitor fell from forklift while being transported, } \\
\text { causing monitor damage }\end{array}$ \\
\hline 95072 & $05 / 25 / 95$ & $\begin{array}{l}\text { Driving forklift across a ditch, caused driver to } \\
\text { jerk, resulted in lumbar strain }\end{array}$ \\
\hline 96073 & $04 / 08 / 96$ & $\begin{array}{l}\text { Developed lower back strain from driving forklift } \\
\text { on bumpy roads }\end{array}$ \\
\hline 96127 & $07 / 19 / 95$ & $\begin{array}{l}\text { Developed discomfort to groin after helping } \\
\text { children up/down on forklift }\end{array}$ \\
\hline \multicolumn{3}{|l|}{$\begin{array}{l}\text { facility } \\
4008002\end{array}$} \\
\hline 92126 & $04 / 14 / 92$ & $\begin{array}{l}\text { Strained back while driving forklift over depression } \\
\text { in floor }\end{array}$ \\
\hline 92138 & $02 / 01 / 92$ & $\begin{array}{l}\text { Van struck parked forklift while van was backing } \\
\text { from building }\end{array}$ \\
\hline 92158 & $07 / 13 / 92$ & $\begin{array}{l}\text { Lacerated leg while driving forklift when forklift } \\
\text { struck Jersey bouncer and overturned }\end{array}$ \\
\hline 92159 & $07 / 13 / 92$ & $\begin{array}{l}\text { Forklift struck Jersey bouncer and overturned } \\
\text { when operator lost control }\end{array}$ \\
\hline 92305 & $11 / 30 / 92$ & $\begin{array}{l}\text { Arm caught between forklift safety cage and shed } \\
\text { gate while entering area }\end{array}$ \\
\hline 93154 & $01 / 19 / 93$ & $\begin{array}{l}\text { Forklift struck against monitor while maneuvering } \\
\text { around concrete column }\end{array}$ \\
\hline 93166 & $07 / 12 / 93$ & $\begin{array}{l}\text { Foot was struck and sprained by airlock doors } \\
\text { while operating forklift }\end{array}$ \\
\hline 93181 & $07 / 19 / 93$ & $\begin{array}{l}\text { Leg was injured when forklift struck employee } \\
\text { standing on side loader }\end{array}$ \\
\hline 93200 & $08 / 23 / 93$ & $\begin{array}{l}\text { Landed on uneven surface and sprained ankle while } \\
\text { descending from forklift }\end{array}$ \\
\hline 93274 & $11 / 19 / 93$ & $\begin{array}{l}\text { Fell to concrete when forklift struck vacuum line, } \\
\text { driver injured head/buttocks }\end{array}$ \\
\hline 94140 & $05 / 17 / 94$ & $\begin{array}{l}\text { Strained lower back when operating forklift for } \\
\text { prolonged period of time }\end{array}$ \\
\hline 94285 & $08 / 09 / 94$ & $\begin{array}{l}\text { Forklift struck and severed transmission line while } \\
\text { entering tank farm }\end{array}$ \\
\hline 94293 & $12 / 28 / 94$ & $\begin{array}{l}\text { Knee struck dash when Cushman vehicle struck } \\
\text { forklift, caused laceration }\end{array}$ \\
\hline
\end{tabular}




\begin{tabular}{|l|l|l|}
\hline $\begin{array}{l}\text { SPMS case } \\
\text { number }\end{array}$ & Event date & One-line description of event \\
\hline $\begin{array}{l}\text { facility } \\
4008002, \\
\text { continued }\end{array}$ & & \\
\hline 95186 & $08 / 11 / 95$ & $\begin{array}{l}\text { Road truck pulled out into intersection and struck } \\
\text { forklift, causing damage }\end{array}$ \\
\hline 95265 & $09 / 05 / 95$ & Machine fell from forklift while being transported \\
\hline 96178 & $09 / 06 / 96$ & $\begin{array}{l}\text { Forklift struck employee, abraded and contused } \\
\text { lower leg, while employee was moving dolly }\end{array}$ \\
\hline 96179 & $09 / 10 / 96$ & $\begin{array}{l}\text { Stepped onto bar on forklift, struck and lacerated } \\
\text { head, shoulder contusion }\end{array}$ \\
\hline $\begin{array}{l}\text { facility } \\
4009006\end{array}$ & & \\
\hline 92002 & $05 / 04 / 92$ & $\begin{array}{l}\text { Split rim tire on forklift recoiled during repair and } \\
\text { caused bruises }\end{array}$ \\
\hline $\begin{array}{l}\text { facility } \\
4009503\end{array}$ & & \\
\hline 93029 & $11 / 18 / 93$ & $\begin{array}{l}\text { Forklift tipped forward while off-loading heavy } \\
\text { magnet, contused knee }\end{array}$ \\
\hline $\begin{array}{l}\text { facility } \\
4516002\end{array}$ & & \\
\hline 93010 & $02 / 15 / 93$ & $\begin{array}{l}\text { Measuring machine fell from skid and was } \\
\text { damaged after transport by forklift }\end{array}$ \\
\hline $\begin{array}{l}\text { facility } \\
4523702\end{array}$ & & \\
\hline 95024 & $06 / 28 / 95$ & $\begin{array}{l}\text { Driver elevated forklift mast, clipped top of } \\
\text { building entrance }\end{array}$ \\
\hline 95049 & $09 / 20 / 95$ & $\begin{array}{l}\text { Forklift struck and damaged concrete base of light } \\
\text { utility pole }\end{array}$ \\
\hline $\begin{array}{l}\text { facility } \\
4523706\end{array}$ & & $\begin{array}{l}\text { Stepped off forklift onto uneven ground after } \\
\text { moving drums, twisting ankle }\end{array}$ \\
\hline 92017 & $1 / 16 / 92$ & Strained back while operating forklift \\
\hline $\begin{array}{l}\text { facility } \\
4539004\end{array}$ & & \\
\hline 93004 & $03 / 04 / 94$ & onarp metal edge of forklift \\
\hline 93025 & $09 / 30 / 95$ & \\
\hline
\end{tabular}




\begin{tabular}{|c|c|c|}
\hline $\begin{array}{l}\text { SPMS case } \\
\text { number }\end{array}$ & Event date & One-line description of event \\
\hline \multicolumn{3}{|l|}{\begin{tabular}{l|l} 
facility \\
6008003
\end{tabular}} \\
\hline 92019 & $04 / 27 / 92$ & $\begin{array}{l}\text { Lost balance and struck/lacerated head on forklift } \\
\text { while standing on boxes }\end{array}$ \\
\hline 94023 & $06 / 20 / 94$ & $\begin{array}{l}\text { Foot was struck and injured after load of steel angle } \\
\text { jarred and fell from forklift skid }\end{array}$ \\
\hline \multicolumn{3}{|l|}{$\begin{array}{l}\text { facility } \\
6009003\end{array}$} \\
\hline 96010 & $09 / 27 / 96$ & $\begin{array}{l}\text { Wind blew forklift door closed which struck } \\
\text { operator's wrist, causing contusion }\end{array}$ \\
\hline \multicolumn{3}{|l|}{$\begin{array}{l}\text { facility } \\
6502001\end{array}$} \\
\hline 92063 & $09 / 01 / 92$ & $\begin{array}{l}\text { Struck and lacerated head on metal cage while } \\
\text { entering forklift }\end{array}$ \\
\hline 93042 & $05 / 20 / 93$ & $\begin{array}{l}\text { Forklift truck struck tailgate of pickup truck while } \\
\text { loading, caused damage }\end{array}$ \\
\hline 94028 & $12 / 21 / 94$ & $\begin{array}{l}\text { Strained lower back when forklift truck struck rail } \\
\text { tracks and immediately stopped }\end{array}$ \\
\hline 94505 & $03 / 23 / 94$ & $\begin{array}{l}\text { Tapchanger was damaged by forklift at unknown } \\
\text { time, causing damage and leakage }\end{array}$ \\
\hline 95067 & $08 / 25 / 95$ & $\begin{array}{l}\text { Knee popped while getting into forklift, causing } \\
\text { injury }\end{array}$ \\
\hline \multicolumn{3}{|l|}{$\begin{array}{l}\text { facility } \\
6509001\end{array}$} \\
\hline 96012 & $07 / 25 / 96$ & $\begin{array}{l}\text { Part tilted off the forklift and broke when it hit the } \\
\text { apron }\end{array}$ \\
\hline \multicolumn{3}{|l|}{$\begin{array}{l}\text { facility } \\
7500503\end{array}$} \\
\hline 95091 & $08 / 28 / 95$ & $\begin{array}{l}\text { Mast of forklift struck and damaged sprinkler } \\
\text { system line }\end{array}$ \\
\hline 96024 & $05 / 13 / 96$ & $\begin{array}{l}\text { Sling slipped off forklift tines and struck head, } \\
\text { causing scalp contusion and abrasion }\end{array}$ \\
\hline \multicolumn{3}{|l|}{$\begin{array}{l}\text { facility } \\
7500605\end{array}$} \\
\hline 95080 & $08 / 24 / 95$ & $\begin{array}{l}\text { Equipment tipped off forklift and onto ground } \\
\text { when forklift tilted }\end{array}$ \\
\hline
\end{tabular}




\begin{tabular}{|c|c|c|}
\hline $\begin{array}{l}\text { SPMS case } \\
\text { number }\end{array}$ & Event date & One-line description of event \\
\hline \multicolumn{3}{|l|}{\begin{tabular}{l|l} 
facility \\
7503004
\end{tabular}} \\
\hline 94126 & $05 / 09 / 94$ & Strained knee while getting in and out of forklift \\
\hline 94129 & $05 / 31 / 94$ & $\begin{array}{l}\text { Strained back while pushing forks together on } \\
\text { forklift }\end{array}$ \\
\hline 94267 & $09 / 28 / 94$ & $\begin{array}{l}\text { Hip was struck and bruised by load when load } \\
\text { shifted during forklift operation }\end{array}$ \\
\hline 96044 & $02 / 27 / 96$ & $\begin{array}{l}\text { Leaned over while operating forklift, causing lower } \\
\text { back strain }\end{array}$ \\
\hline 96114 & $05 / 28 / 96$ & $\begin{array}{l}\text { Struck and lacerated knuckle on shim while putting } \\
\text { bearing on forklift }\end{array}$ \\
\hline \multicolumn{3}{|l|}{$\begin{array}{l}\text { facility } \\
7509002\end{array}$} \\
\hline 92062 & $03 / 11 / 92$ & $\begin{array}{l}\text { Strained muscle in knee while dismounting from } \\
\text { forklift }\end{array}$ \\
\hline 92153 & $08 / 15 / 92$ & $\begin{array}{l}\text { Struck head against safety cage of forklift, causing } \\
\text { laceration }\end{array}$ \\
\hline 93039 & $02 / 10 / 93$ & $\begin{array}{l}\text { Tooth was fractured by buckle while removing } \\
\text { safety belt from forklift }\end{array}$ \\
\hline 93080 & $03 / 02 / 93$ & $\begin{array}{l}\text { Slipped on icy running boards and strained lower } \\
\text { back while exiting forklift }\end{array}$ \\
\hline 93305 & $09 / 15 / 93$ & Fractured leg while jumping from moving forklift \\
\hline 93382 & $12 / 17 / 93$ & $\begin{array}{l}\text { Slipped and twisted/strained back while attempting } \\
\text { to install forklift door }\end{array}$ \\
\hline 95186 & $08 / 10 / 95$ & $\begin{array}{l}\text { Driving forklift and moving cabinet, caused lower } \\
\text { back strain }\end{array}$ \\
\hline 96075 & $04 / 25 / 96$ & Developed hand strain from driving forklift \\
\hline \multicolumn{3}{|l|}{$\begin{array}{l}\text { facility } \\
7509004\end{array}$} \\
\hline 93053 & $01 / 20 / 93$ & $\begin{array}{l}\text { Pickup truck bumper was damaged when forklift } \\
\text { slid downhill while being towed }\end{array}$ \\
\hline 93094 & $04 / 24 / 93$ & $\begin{array}{l}\text { Forklift mast was damaged while forklift was being } \\
\text { pulled by truck }\end{array}$ \\
\hline \multicolumn{3}{|l|}{$\begin{array}{l}\text { facility } \\
7711004\end{array}$} \\
\hline 93021 & $02 / 01 / 93$ & $\begin{array}{l}\text { Slipped on gravel and sprained knee while getting } \\
\text { out of forklift }\end{array}$ \\
\hline 93078 & $02 / 11 / 93$ & $\begin{array}{l}\text { Strained shoulder while maneuvering stiff steering } \\
\text { wheel on forklift }\end{array}$ \\
\hline 94072 & $04 / 07 / 94$ & $\begin{array}{l}\text { Crate fell from moving forklift and struck toe, } \\
\text { causing fracture }\end{array}$ \\
\hline
\end{tabular}




\begin{tabular}{|c|c|c|}
\hline $\begin{array}{l}\text { SPMS case } \\
\text { number }\end{array}$ & Event date & One-line description of event \\
\hline \multicolumn{3}{|l|}{\begin{tabular}{l|l} 
facility \\
7712002 \\
\end{tabular}} \\
\hline 94003 & $08 / 02 / 94$ & $\begin{array}{l}\text { Handling bar struck/contused top of foot when } \\
\text { spacer plate slid from forks }\end{array}$ \\
\hline \multicolumn{3}{|l|}{$\begin{array}{l}\text { facility } \\
8003004\end{array}$} \\
\hline 93048 & $09 / 10 / 93$ & $\begin{array}{l}\text { Experienced back pain while climbing up and down } \\
\text { forklift }\end{array}$ \\
\hline 94054 & $10 / 07 / 94$ & $\begin{array}{l}\text { Forklift mast fell and crushed fingers while } \\
\text { adjusting restraints }\end{array}$ \\
\hline 95357 & $10 / 11 / 95$ & $\begin{array}{l}\text { Stepped off uneven pavement while exiting forklift, } \\
\text { causing unknown injury }\end{array}$ \\
\hline \multicolumn{3}{|l|}{$\begin{array}{l}\text { facility } \\
8004003\end{array}$} \\
\hline 94248 & $08 / 10 / 94$ & $\begin{array}{l}\text { Forklift tipped over and mast struck/lacerated head, } \\
\text { required sutures }\end{array}$ \\
\hline 95112 & $06 / 12 / 95$ & $\begin{array}{l}\text { Developed lower back strain from climbing on and } \\
\text { off forklift }\end{array}$ \\
\hline 95159 & $08 / 18 / 95$ & $\begin{array}{l}\text { Employee strained back while removing drive } \\
\text { wheel from forklift }\end{array}$ \\
\hline 95288 & $06 / 01 / 95$ & $\begin{array}{l}\text { Jumped off moving forklift while securing truck } \\
\text { load, caused twisted knee }\end{array}$ \\
\hline 96168 & $07 / 18 / 96$ & $\begin{array}{l}\text { Struck knee on gas filler cap while exiting forklift, } \\
\text { multiple injuries }\end{array}$ \\
\hline \multicolumn{3}{|l|}{$\begin{array}{l}\text { facility } \\
8008003\end{array}$} \\
\hline 92002 & $01 / 22 / 92$ & $\begin{array}{l}\text { Portable grinder recoiled during repair of forklift } \\
\text { and lacerated finger }\end{array}$ \\
\hline 94050 & $05 / 26 / 94$ & $\begin{array}{l}\text { Pipes struck against pickup truck when being } \\
\text { moved to salvage by forklift }\end{array}$ \\
\hline 95006 & $01 / 31 / 95$ & $\begin{array}{l}\text { Strained lower back when brakes locked up while } \\
\text { driving forklift }\end{array}$ \\
\hline 95025 & $06 / 06 / 95$ & $\begin{array}{l}\text { Employee was exposed to propane exhaust, caused } \\
\text { shortness of breath }\end{array}$ \\
\hline 95072 & $08 / 19 / 95$ & $\begin{array}{l}\text { Driver was unloading fork truck, load fell, } \\
\text { damaging unit and truck }\end{array}$ \\
\hline 95075 & $05 / 11 / 95$ & $\begin{array}{l}\text { Unattended forklift rolled and struck pickup truck, } \\
\text { causing damage to pickup }\end{array}$ \\
\hline
\end{tabular}




\begin{tabular}{|c|c|c|}
\hline $\begin{array}{l}\text { SPMS case } \\
\text { number }\end{array}$ & Event date & One-line description of event \\
\hline \multicolumn{3}{|l|}{$\begin{array}{l}\text { facility } \\
8500505\end{array}$} \\
\hline 92049 & $04 / 27 / 92$ & $\begin{array}{l}\text { Load of pipe fell off forklift tines and struck } \\
\text { shoulder, causing contusion }\end{array}$ \\
\hline 94130 & $11 / 09 / 94$ & $\begin{array}{l}\text { Ring caught on window track and lacerated finger } \\
\text { while dismounting forklift }\end{array}$ \\
\hline \multicolumn{3}{|l|}{$\begin{array}{l}\text { facility } \\
8501002\end{array}$} \\
\hline 92024 & $03 / 05 / 92$ & $\begin{array}{l}\text { Forklift fell from dock opening and crushed foot, } \\
\text { resulting in amputation }\end{array}$ \\
\hline 92034 & $03 / 05 / 92$ & $\begin{array}{l}\text { Forklift fell off dock while being backed, causing } \\
\text { minimal equipment damage }\end{array}$ \\
\hline 93123 & $10 / 22 / 93$ & $\begin{array}{l}\text { Banding draped across forklift spring loose and } \\
\text { struck/lacerated arm }\end{array}$ \\
\hline \multicolumn{3}{|l|}{$\begin{array}{l}\text { facility } \\
8501014\end{array}$} \\
\hline 93001 & $03 / 09 / 93$ & $\begin{array}{l}\text { Forklift knocked drum into lower abdomen while } \\
\text { turning, causing contusion }\end{array}$ \\
\hline 94026 & $04 / 20 / 94$ & $\begin{array}{l}\text { Bracket swung down and contused forehead while } \\
\text { installing fuel system on forklift }\end{array}$ \\
\hline \multicolumn{3}{|l|}{$\begin{array}{l}\text { facility } \\
9005003\end{array}$} \\
\hline 94023 & $09 / 15 / 94$ & $\begin{array}{l}\text { Boxes being transported on forklift fell off and } \\
\text { struck/damaged vehicle }\end{array}$ \\
\hline \multicolumn{3}{|l|}{$\begin{array}{l}\text { facility } \\
9508003\end{array}$} \\
\hline 92019 & $02 / 26 / 92$ & $\begin{array}{l}\text { Strained back while stepping off forklift rack while } \\
\text { carrying table }\end{array}$ \\
\hline 92032 & $03 / 30 / 92$ & $\begin{array}{l}\text { Forklift struck against private vehicle while being } \\
\text { backed from building }\end{array}$ \\
\hline 93110 & $09 / 21 / 93$ & $\begin{array}{l}\text { Lacerated finger on metal guard while attempting to } \\
\text { exit forklift }\end{array}$ \\
\hline 94095 & $08 / 30 / 94$ & $\begin{array}{l}\text { Repositioning pallet with forklift, pallet tilted and } \\
\text { fell, causing damage }\end{array}$ \\
\hline \multicolumn{3}{|l|}{$\begin{array}{l}\text { facility } \\
9606508 \\
\end{array}$} \\
\hline 93010 & $02 / 24 / 93$ & $\begin{array}{l}\text { Forklift mast struck partially open roll-up door } \\
\text { during forklift entry into building }\end{array}$ \\
\hline 94039 & $09 / 19 / 94$ & $\begin{array}{l}300 \text { pound valve shifted and fell from forklift and } \\
\text { struck employee's toe, injury resulted in surgery }\end{array}$ \\
\hline 94054 & $11 / 18 / 94$ & $\begin{array}{l}\text { Fractured fingers between fork bar and boom } \\
\text { housing while lifting a pallet }\end{array}$ \\
\hline
\end{tabular}


Scanning the DOE data reveals several new issues and confirms others. Not only were there operations injuries, but maintenance injuries as well. This report focuses only on operations events. Driver mounting and dismounting injuries were numerous, along with falling or shifting loads, and lift trucks colliding with an object or a person. Drivers suffered from shocks of driving over uneven ground, there were several pinch point accidents, two events of wheels throwing objects, a few lift truck rollover events, and several events where lift truck drivers or other workers hit their head by falling and striking a lift truck as they fell. Several types of events found in this data set were not foreseen. The first unforeseen type is where the drivers of lift trucks get foreign objects in their eyes. This event should have been foreseeable since the lift trucks often pick loads from heights and that action can cause dust and particles to leave surfaces, thus becoming suspended in the air. Driving forward with a load can cause small air currents on the load, further dislodging dust and allowing the driver to drive into this dust. Another unforeseen event was recorded as a driver being bitten by an insect while driving the lift truck (fortunately, there was no unfortunate consequence because of the driver's distraction). The next type of unforeseen event was a hydraulic line break releasing hydraulic fluid onto the lift truck driver, and the event where the rear wheel broke off from the lift truck was not one of the anticipated failures of the lift truck. Two events discussed drivers leaving a moving lift truck. There was one event where the lift truck brakes failed in the locked on position while the lift truck was moving (and one event where the parking brake failed off, allowing the lift truck to roll away), and there were also two tine failures recorded over the time period. This set of events is considered to be a typical example of the lift truck-related events that can occur in industry.

Stout-Wiegand (1987) discusses forklift related injuries in the US. In the 1980's, there were up to 34,000 injuries per year related to lift trucks. Drivers had strained backs, pinched extremities, contusions, and injuries from falls. Other workers suffered from being run over (i.e., toes run over) by lift trucks, being caught between trucks and other objects, being struck by lift trucks, and other injuries. Viewing Stout-Wiegand's data and National Safety Council (NSC, 1995) data shows that there has been a decrease in worker injuries over the period from 1984 to 1992 by roughly a factor of 2 . Reasons for this decrease are not evident. Potential reasons could be economic (less lift truck usage with a slowing economy), regulatory (OSHA implementing rules about lift trucks), or enhanced safety awareness within companies (e.g., voluntary protection programs).

The Federal Register (FR, 1995) also gives some statistics about forklift injuries in the commercial sector. In a discussion about increasing operator training requirements, actuarial data on lift truck accidents was used to point out the areas needing more work. For 1991-1992, 41 operators died when their lift truck overturned, 13 died when the lift truck struck something or ran off of a dock, 19 workers died when pinned between the lift truck and an object, 29 workers died when struck by falling materials, 24 workers died when struck by lift trucks, 24 workers fell from forklifts (either as passengers on the truck body or from riding the tines), 10 workers died during forklift repair operations, and 10 workers died from other accidents related to lift trucks.

Factory Mutual data (FMR, 1993) states that lift truck losses in the US between 1987-1992 were over $\$ 160$ million, and $\$ 146$ million of that was fire. The most severe losses were 
when lift trucks were carrying flammable liquid containers. When the load was inadvertently dropped, the flammable liquids spilled and were ignited by the hot surfaces of the lift truck or sparks from the lift truck. An electric-powered lift truck has been assumed to be the ignitor for a large warehouse fire in June 1982, where a box of aerosol cans fell and some cans ruptured, releasing vapors that ignited to cause an intense fire (FMR, 1993; NFPA, 1983). Other events contributing to the $\$ 160$ million loss were water damage from broken piping and repairs to structural damage from lift truck impact events.

Literature Review of Lift Truck Hazards. To provide more insight into the hazards analysis, a literature review of lift truck hazards was completed. Several safety texts discuss lift truck hazards. Brauer (1994) gives several guides for materials handling safety. He states that this equipment has visibility hazards, which lead to possible collisions with people or structures. All lift trucks have traffic hazards and they also have minimum space requirements for movement. Lift trucks have load limits; exceeding these limits can lead to structural failure. Brauer also states that many lift trucks have high centers of gravity so that when these trucks are operated too fast in a turn, they can roll over. This effect is even more pronounced when a load is being elevated during a sharp turn. Brauer also stated that a classic products liability case involved a materials handling vehicle that was not equipped with a rear view mirror.

Asfahl (1995) also discusses lift truck hazards. Asfahl discusses the lift truck classifications defined in the Code of Federal Regulations, and meeting the spirit if not the letter of the law in the lift truck approval process. While many rules focus on lift truck fires and explosions, Asfahl warns that lift truck operations, fueling, guarding, operator training, and maintenance are also important to a lift truck safety program. Some of the safety advice is for electric battery powered trucks: ignitable gases are evolved during battery charging, and sparks are often formed when battery connections are made - so good ventilation is essential for safety as well as an eyewash and emergency shower station.

Asfahl (1995) also discusses internal combustion engine trucks; these all generate carbon monoxide gas in their exhaust. General ventilation of the work area must be adequate to reduce carbon monoxide levels to the threshold limit value (50 parts per million) or below to provide for worker safety. Other issues to review are if the drivers allow the engines to remain on when not necessary, can the lift truck paths be changed to reduce carbon monoxide buildup in the buildings, and are the lift trucks in proper tune to produce the lowest emissions. He also points out that lift truck lights are required by the Occupational Safety and Health Administration if lighting is less than 2 lumens per square foot (for comparison, an office building is usually about 100 lumens per square foot). Asfahl also states that one of the greatest hazards with forklifts is the transition from the dock to the road trailer or rail car. He shows proper usage of wheel chocks and dockboards, including slow lift truck movement to avoid vibrating or bouncing the dockboard out of position. He cites lift truck stability as a problem, and driver visibility as another problem. Asfahl also discusses the frequent although illegal use of empty pallets as platforms for elevating workers on the forks. He explains that small obstructions encountered in raising the platform could damage the pallet, tip the pallet, injure the worker riding up on the pallet, or knock the worker off balance and risk a fall from height. 
Asfahl (1995) also discusses operator training. He states that lift trucks are unlike automobiles since lift trucks have a much shorter wheel base, have a high center of gravity which leads to poor stability, and have small diameter wheels (which compound the stability problem). Lifting and placing loads requires skill that usually comes from good training. Maintenance of the lift truck is also important. Lift trucks must be inspected for safety daily, or if used in three shift operation then safety inspections are required after each shift. In contrast, motor vehicles (i.e., automobiles and light utility vehicles) are only usually required to be inspected for safety once a year. There is no latitude is permitted by the US Occupational Safety and Health Administration to allow defective lift trucks to continue to be operated until a regular maintenance interval or overhaul. Any condition, such as a bad horn, etc., is enough cause to remove the lift truck from service for repair.

Lovested (1977) gives the top ten lift truck accidents. By his definition, these accident categories are: struck by forklift; struck by object; operator hit by falling object; other employee hit by object; boarding or leaving truck incidents; truck nearly tips over; collision with other vehicles; fork lift falling off of loading dock; part of body hit by object; and parts falling onto operator. This work was published before falling object protective structures (FOPS) were required for indoor lift trucks. Lovested's data showed that mechanical failures caused less than $1 \%$ of lift truck accidents.

Taub (1988) discusses some practical means of reducing lift truck collisions. He states that common lift truck damage reports include bent pallet racks, cracked walls and columns, smashed raw materials, splintered doors, dented electrical panels, bent or dented pipes, dented or torn ventilation ducts, and damaged fire protection equipment. About $80 \%$ of lift truck damage is caused by drivers working under strict time constraints and carrying heavy loads. Taub states that even in an ideal plant environment, an operator hits a structural member or piece of equipment about once a month. He recommends using low barriers embedded in the floor as a practical and economical approach for protecting equipment. The lift truck cannot climb over $15 \mathrm{~cm}$-tall obstacles, and they have low ground clearance. Another tactic to protect overhead areas is the use of clangers. These are roughly 1-m long pieces of concentric diameter pipes sleeved together, hanging from overhead areas so that when a load is lifted up or is being moved while elevated, the load strikes the clanger and the pipes make a 'clanging' noise. The clanger noise alerts the operator to the height of the load and the operator must then prevent the load from hitting a ceiling beam, cable trays, lighting, fire sprinkler piping, ventilation ducts, or any other overhead equipment.

Conclusions. The energy based hazards analysis appears to have practical completeness in that anything which might have been overlooked is not consequential. There are many hazards in using lift trucks, but we must retain perspective. Manual material handling and other systems (robot vehicles, conveyor belts, etc.) have not been evaluated for a comparison of hazards because that task is large and outside the scope of this report. Surprisingly, a few authors still recommend manual handling to avoid lift truck costs (Cecala et al., 1989). After hazard identification, the next step is hazard control. If lift truck hazards are managed properly, the benefits of lift truck productivity can be enjoyed while the liabilities of industrial accidents can be precluded or minimized. The next chapter discusses regulations, consensus standards, and other guidance to help control lift truck hazards. 


\section{Chapter 3 References}

Asfahl, 1995.

Booth, 1979.

Brauer, 1994.

Cecala et al., 1989.

Cole, 1992.

DOE ESH, 1992.

FMR, 1993.

FR, 1995.

Johnson, 1980.

Kletz, 1988.

Larsson and Rechnitzer, 1994.
C. R. Asfahl, Industrial Safety and Health Management, third edition, Prentice Hall, Englewood Cliffs, New Jersey, ISBN 0-13-140881-X, 1995, Chapter 12.

R. T. Booth, "Making Factories Safe for Fork Lift Truck Drivers," Occupational Health, April 1979, p. 193-197.

R. L. Brauer, Safety and Health for Engineers, Van Nostrand Reinhold, New York, ISBN 0-442-01856-8, 1994, chapter 15.

A. B. Cecala et al., "Reducing Workers' Dust Exposure during Bag Stacking in Enclosed Vehicles," American Industrial Hygiene Association Journal, 50, 1989, pages 99-104.

L. S. Cole, The Investigation of Motor Vehicle Fires, third edition, Cole Books, Novato, California, ISBN 0939818-22-1, 1992, pages 154-155.

"Worker Safety Compromised When Operating Forklifts in Reverse," Environment, Safety \& Health Bulletin, DOE/EH-0255, US DOE, Issue 92-3, June 1992.

"Lift Trucks", Factory Mutual Record, 70, March/April 1993, pages 3-8.

US Federal Register, Part II, Department of Labor, Occupational Safety and Health Administration, Powered Industrial Truck Operator Training: Proposed Rule, volume 60, number 49, Tuesday, March 14, 1995, pages $13782-13831$.

W. G. Johnson, MORT Safety Assurance Systems, Marcel Dekker, Inc., New York, ISBN 0-8247-6897-3, 1980 , pages 37-39.

T. A. Kletz, Learning from Accidents in Industry, Butterworths, London, UK, ISBN 0-408-02696-0, 1988, page 125 .

T. J. Larsson and G. Rechnitzer, "Forklift trucks analysis of severe and fatal occupational injuries, critical incidents and priorities for prevention," Safety Science, 17, 1994 , pages $275-289$. 
Lovested, 1977.

Marryatt, 1988.

NFPA, 1972.

NFPA, 1983.

NSC, 1995.

O'Mara, 1989.

OSO, 1993.

OSHA, 1996.

Stout-Wiegand, 1987.

Taub, 1988.

Williams and Priestly, 1980.
G. E. Lovested, "Top Ten Fork-Lift Truck Accidents," National Safety News, 116, Sept. 1977, pages 123-127.

H. W. Marryatt, Fire: A Century of Automatic Sprinkler Protection in Australia and New Zealand. 1886-1986, National Fire Protection Association, Quincy, Massachusetts, ISBN 0-7316-4001-2, 1988

C. B. Patterson, "Powered Industrial Trucks: Appraising Their In-Plant Fire Safety," Fire Journal, 66, September 1972, pages 103-104.

R. Best, "\$100 Million Fire in K Mart Distribution Center, Sprinkler Systems Overwhelmed," Fire Journal, 77, March 1983, pages 36-42.

National Safety Council, Accident Facts, 1995 edition, NSC, Itasca, Ilinois, ISBN 0-87912-185-8, 1995.

N. O'Mara, A Study of Fork Lift Truck Accidents in South Australia, South Australian Health Commission, ISBN 0-7243-4027-0, February 1989.

"Worker Struck by Forklift," DOE Occupational Safety Observer, 2, November 1993, page 1-2.

"OSHA Says Death of Worker Shows Dangers of Unauthorized Modification of Industrial Forklift Trucks," news release USDL 96-278, United States Department of Labor, Occupational Safety and Health Administration, July 11, 1996. Also see the internet address http://www.osha.gov/media/oshanews/.

N. Stout-Wiegand, "Characteristics of Work-Related Injuries Involving Forklift Trucks," Journal of Safety Research, 18, 1987, pages 179-190.

R. S. Taub, "Protecting Structures and Equipment from Lift Truck Damage," Plant Engineering, 42, October 13, 1988, pages 112-114.

E. A. Williams and S. E. Priestly, "Fork Lift Truck Injuries," Journal of the Society of Occupational Medicine, 30, 1980, pages 149-152. 


\section{Regulations and Standards for Lift Trucks}

This chapter provides an overview of lift truck regulations, standards, guidelines, and recommended practices from the government and other sources, such as engineering societies and industrial groups. The chapter is arranged in the following order: Code of Federal Regulations, Underwriters Laboratories, National Fire Protection Association, American National Standards Institute/American Society of Mechanical Engineers, Society of Automotive Engineers, Factory Mutual, National Safety Council, and the US Department of Energy.

Code of Federal Regulations. In Title 29, Labor, of the Code of Federal Regulations there is a section addressing powered industrial trucks (CFR, 1996). This section is a set of regulations for lift truck usage. The lift truck regulations cover safety in operations, maintenance, operator training, and fire protection. Some lift truck safety apparatus is also specified in this set of regulations. Non-compliance is punishable by law. This set of regulations is the most broad of those located for this report. This set of rules is covered in detail since it is law, while other industrial standards are usually voluntary compliance.

The types of lift trucks are specified to be marked on the lift truck - D, DS, DY, E, ES, EE, EX, G, GS, LP, and LPS. The D designators are diesel powered lift trucks (D), diesel with safeguarded exhaust systems to prevent any sparks or backfires from igniting any explosive substances (gas, dust, etc.) in the atmosphere (DS), and diesel with safeguards and no electrical equipment and a temperature limitation feature (DY) (to avoid autoignition of gas or dust explosions). The E designators are electric battery powered lift trucks (E) with minimum safeguards against inherent fire hazards, electric powered trucks with safeguards against sparks and limitations of surface temperatures (ES), electric powered lift trucks with the $E$ and $E S$ ratings that also have the motor and other electrical equipment completely enclosed (EE), and electric powered trucks that are constructed to enter into atmospheres containing flammable vapors or dusts (EX). The gasoline powered units (G) have safeguards against basic, inherent fire hazards. The LP designation is similar to $G$ except that liquefied petroleum gas (LP gas, mainly consisting of propane) is used instead of gasoline. LPS is a designator for an LP gas powered lift truck that has safeguards on the exhaust, fuel and electrical systems to prevent ignition of any hazardous vapors or dusts in the atmosphere. There are tables to show which designations of lift trucks can be used in the presence of which gases, dusts (metal, agricultural, and industrial), and fibers.

Safety equipment is specified for lift trucks, including overhead guards for the cab of high lift trucks and a vertical load backrest extension so that loads cannot topple backward onto the lift truck cab. Fuels must be handled according to the National Fire Protection Association standard 30 (Flammable and Combustible Liquids Code, 1969 version) or standard 58 (Liquefied Petroleum Gases, 1969 version). There are rules about battery charging areas to mitigate any discharging hydrogen gas from batteries and to neutralize any acid spills. When lift trucks are operating in areas where lighting levels are below 2 lumens per square foot, the lift truck shall have auxiliary lighting. 
Operator training is specified in this regulation. Only trained and authorized operators are allowed to use lift trucks. Specifications for depth of training or periodic renewal are not given.

Lift truck operations are also regulated. Lift trucks shall not be driven up to persons standing in front of a bench or other fixed object (presumably to preclude trapping a person between the truck and the fixed object). No person shall be permitted to stand or pass beneath the elevated portion of a lift truck whether it is loaded or empty. The reason for this rule is clear. As described in the previous chapter, in 1996 there was a fatality when a load detached and fell onto a worker. Unauthorized persons shall not ride on powered industrial trucks. If riding is authorized, then a safe place to ride on the lift truck shall be provided. This rule means that workers should not ride by standing on the forks and they should not perch themselves on parts of the lift truck body unless a seat has been provided for that purpose, because several fatalities have occurred by falling from the lift truck. When a lift truck is unattended (the driver is $7.65 \mathrm{~m}$ [25 feet] or further away from the truck, or the driver is out of sight of the lift truck) then the lift truck controls should be neutralized, power shut off, load on the ground (if there is no load, then the forks must be lowered to the ground), and the wheels blocked if the truck is on an incline. This rule is meant to preclude unauthorized persons from using the lift truck. It is a safety rule, but many would argue about its viability in the workplace due to its inconvenience.

There are more safety rules in the CFR. The lift truck shall maintain a safe distance from the edge of a dock, elevated platform or rail car. Lift trucks shall not be used for opening and closing freight doors. When loading or unloading road trucks, the road truck's trailer shall be secured from moving or 'see-sawing' and the floor integrity shall be checked. Brodbeck (1996) explains that lift trucks have fallen through road trailer floors and these events have injured, and even killed, lift truck operators. When a road trailer is loaded and the several ton lift truck also enters the trailer, overloads are possible.

Lift trucks shall have sufficient headroom beneath overhead installations (lights, air ducts, fire sprinklers, piping, etc.). This rule is not always observed when a lift truck is purchased for an existing facility.

An overhead guard is to be provided to protect against falling objects (this is the so-called falling object protection system, or FOPS). This system is intended to protect against small falling objects, not to protect against a truck capacity load from toppling onto the lift truck cab. A backrest extension shall be used when necessary to minimize the possibility of the load or part of the load from falling backward. Therefore, the FOPS protects against a unit (for example, a sack from a pallet) falling back onto the driver, and the backrest extension protects the driver from the entire load falling back toward the cab.

If personnel are to be elevated by the lift truck, a safety platform shall be used. Such a platform is pictured in Laing (1992). This platform has guardrails to prevent falls from height. Too often in industry, only an empty pallet is used as a working platform; the deck of this platform is not uniform so footing can be precarious. The pallet, of course, has no hand rails. 
When traveling, the lift truck must obey plant speed limits and keep a safe distance of three truck lengths between it and all other trucks in the area. Lift trucks shall yield to ambulances, fire engines, and other emergency vehicles. Lift trucks traveling the same direction shall not pass each other at intersections, blind spots, or other unsafe locations. The lift truck driver shall slow down and honk the horn at intersections and other locations where vision is obstructed. The driver is required to look in the direction of travel and keep a clear view of the path of travel. One of the examples above showed that the driver did not watch the direction of travel and struck a person. Inclines or grades shall be ascended or descended slowly (to avoid picking up speed if brakes were to lose effectiveness or fail). When descending or ascending grades over $10 \%$ slope, loaded trucks shall be driven with the load upgrade. This is suggested for stability of the lift truck. On all grades, the load shall be tilted back if applicable, and the load raised only far enough to clear the road surface. The lower the load is kept, the lower the center of gravity of the lift truck and load system, so the lift truck is more stable (i.e., more resistant to tipping over).

Stunt driving and horseplay shall not be permitted (literature review shows that this rule has been violated). Drivers are also required to slow down for wet or slippery floors. Perhaps this is one of the rules wherein familiarity allows the drivers to believe that the hazard is less severe than they are told. Nonetheless, the lift truck can be unstable and can topple if it skids sideways, and like an automobile the braking distance is increased on slick surfaces.

Dockboards or bridge plates shall be properly secured before being driven upon. Brodbeck (1996) discusses reasons for such a rule. If this bridge between a dock and a road trailer is at all loose, there is a hazard that the lift truck could fall from the dock height to the ground. Another rule is that elevators shall be approached slowly and entered squarely (probably to avoid striking the side walls of the elevator shaft). On the elevator the lift truck operator is supposed to neutralize the controls, turn off the motor, and set the brakes. This rule has likely arisen from a combination of several issues. Turning off the motor in a confined space will keep the operator from breathing high concentrations of exhaust gases and from having the elevator space warm up from exhaust gases. Lowering the load and setting the brakes prevents the lift truck from shifting. Since an elevator ride may only last a few minutes, perhaps this rule is not always followed.

Running over loose objects on the roadway surface shall be avoided. This rule is directed at housekeeping, in the case that the lift truck wheels could throw objects or that the objects could damage a tire enough to cause the operator to lose control of the lift truck. This is but one of many reasons to have good housekeeping in the plant environment. Other leading reasons for plant cleanliness are fire safety, employee hygiene, and reduction in contamination of the product.

While negotiating turns, speed shall be reduced by means of turning the hand steering wheel in a smooth, sweeping motion. The hand steering wheel shall be turned at a moderate, even rate unless moving very slowly. This rule is given to preclude sharp turns at high speeds. The lift truck will make such turns, but only with a high likelihood of tipping over. Many authors warn against sharp turns at high speed. 
Only stable and arranged loads shall be handled. Caution shall be used when handling offcenter loads that cannot be stabilized. Do not lift loads greater than the capacity of the lift truck. Lift trucks equipped with special attachments (such as a drum handler, a box stacker, etc.) shall be treated as partially loaded even when not handling a load. The mast shall be tilted backward to stabilize the load. Only enough backward tilt to stabilize the load shall be used. These rules protect the driver and other workers from loads falling off pallets, fork failure from overloading, and providing load stability.

If at any time the lift truck is found to be in need of repair, or if it is found to be defective in any way, it shall be removed from service until it has been restored to safe operating condition. Fuel tanks shall not be filled with the engine running. Spillage of fuel or oil shall be carefully washed away or evaporated and the fuel cap replaced before starting the engine. No lift truck shall be operated with a leak in the fuel system until after the leak has been repaired. No open flames shall be used to check battery electrolyte level or gasoline level.

Any lift truck not in safe condition shall be removed from service and repaired by authorized personnel. No repairs shall be made in Class I, II, or III locations (NFPA 505 discusses these classifications; basically, Class I may have flammable vapors, Class II may have combustible dusts, and Class III may have ignitable fibers in the air). Replacement parts must be equivalent to original equipment. Additional counterweighting of a lift truck must be approved by the manufacturer. Industrial trucks shall be examined daily for any conditions that adversely affect safety. If the lift truck is operated on a round-the-clock basis, then it shall be examined after each shift.

Lift trucks with mufflers having screens or other parts that may become clogged shall not be operated until such screens are cleaned. Any vehicle that emits sparks or flames from the exhaust shall be removed from service immediately, and cannot return to service until the cause of sparks or flames has been eliminated. If the lift truck has any part that is overheating, it must be removed from service until the cause for overheating has been eliminated. Lift trucks shall be kept in clean condition, free of lint, excess soil or grease, or other combustibles. Non-combustible agents shall be used to clean lift trucks. Only high flash point solvents (that is, over $38^{\circ} \mathrm{C}$ or $100^{\circ} \mathrm{F}$ ) may be used.

There is another section on lift trucks in the CFR (CFRa, 1996). In the construction section, there are some rules about lift truck use. The rated capacity will be displayed for the operator, and shall not be exceeded. No modifications or additions that affect the capacity or safe operation of the vehicle shall be made without the manufacturer's written permission. Loads lifted by two or more lift trucks must be balanced so that no truck is overloaded. Steering wheel knobs for quick wheel turning shall not be used unless the steering mechanism prevent road reactions from moving the steering wheel. All high lift trucks must have an overhead guard that meets requirements in ANSI B56.1-1969. All industrial trucks shall meet the applicable requirements in ANSI B56.1-1969. There shall be no riders on lift trucks unless a safe place has been made for them. Lift trucks that can elevate people must have a safety platform that can be firmly secured to the lifting carriage 
and/or forks, a means for people on the platform to shut off power to the lift truck, and overhead protection from falling objects if the work conditions warrant such protection.

The preceding summary of the US Occupational Safety and Health Administration (OSHA) rules for lift trucks does not mention several items, such as using natural gas as a fuel for lift trucks, carrying a fire extinguisher aboard the vehicle, having an engine fire suppression system, having driver seat belts, and other safety issues discussed in Chapter 5. Perhaps some of these items are too expensive to justify from a risk reduction viewpoint, as in a cost-benefit analysis. Two interesting articles about OSHA rules for lift trucks are Mruk (1973) and Evans (1979).

Underwriters Laboratories. There are two safety standards for powered industrial trucks, one for electric powered trucks (UL 583, 1991) and one for internal combustion engine-powered trucks (UL 558, 1991). The electric powered truck standard will be discussed first.

Electric powered lift trucks. UL standard 583 (1991) specifies electrical equipment for lift trucks. Motors, controls, wiring, batteries, leads, connectors, lamps, circuits, and other equipment for type E, EE, and EX lift trucks. Maximum temperatures for equipment under test conditions are given. For example, a brake test of type $E$ lift trucks is a test unit being brought to a stop every $15 \mathrm{~m}$ (50 feet), continuing until constant temperatures of the external surfaces of brakes are reached. A constant temperature is considered to be reached if over one hour of testing the temperature does not fluctuate more than $3^{\circ} \mathrm{C}\left(5^{\circ} \mathrm{F}\right)$. The brake external surface temperatures must not exceed $175^{\circ} \mathrm{C}\left(347^{\circ} \mathrm{F}\right)$ when the ambient temperature in the room is $25^{\circ} \mathrm{C}\left(75^{\circ} \mathrm{F}\right)$. This standard is intended for setting criteria for lift trucks to be mechanically and electrically robust. Issues of the lift truck catching fire or igniting an explosion are discussed, and there are provisions for operator protection from fire. Some of the provisions for electrical insulation also serve to protect operators and maintainers from electrocution. The type EX lift truck has the most demanding requirements for suppressing any ignition of explosions in flammable atmospheres. For example, there can be no belt drives in this lift truck unless the belts are electrically conductive or are enclosed to avoid static electricity buildup or belt slippage causing heating that could ignite Class I, group D explosive atmospheres. The battery must be covered with an inert gas under pressure or covered with insulation or protected from possible contact with foreign objects and ambient gases or vapors when the battery cover is down. Wheels and tires for type EX lift trucks must be non-sparking, and two of the wheels shall be electrically conductive or have a static discharge device. The resistance of a nonmetallic conductive wheel or tire shall not exceed 250,000 ohms.

Internal combustion lift trucks. UL 558 (1991) discusses design and testing for systems used for internal combustion engine lift trucks. This standard outlines requirements for electrical systems, exhaust systems, fuel systems, and tests for safety. The safety tests include backfire tests, muffler tests, exhaust system tests, and LP gas container tests. Lift trucks of types G, GS, D, DS, DY, LP, and LPS are discussed. Basic safety ideas are discussed, such as using fuses in electrical circuits, making design provisions to avoid chafing electrical insulation, maintaining wiring clearance from hot parts, keeping hot parts 
free of oil, fuel, and grease, and other ideas. Fuel containers must be constructed in accordance with the American Society of Mechanical Engineers Boiler and Pressure Vessel Code, with a working pressure of $2.6 \mathrm{MPa}(375 \mathrm{psig})$, have a volume of $0.95 \mathrm{~m}^{3}$ ( 1.5 cubic feet) or less, and be able to withstand a pressure 5 times the marked working pressure or, for liquefied petroleum gas, the fuel tank is built to US Department of Transportation rules, marked for minimum service pressure of $1.7 \mathrm{MPa}$ (240 psig). The gas fuel container shall have a safety relief valve. Fuel tanks shall have sufficient capacity for an 8 hour operating period.

Maximum temperatures during operation can be:

electrical insulation $\quad 150^{\circ} \mathrm{C}\left(270^{\circ} \mathrm{F}\right)$

gasoline or diesel fuel in tank $50^{\circ} \mathrm{C}\left(90^{\circ} \mathrm{F}\right)$

brake external surfaces $175^{\circ} \mathrm{C}\left(347^{\circ} \mathrm{F}\right)$

UL 558 is intended mainly as a set of sound practices in design and construction of lift trucks to make them fire safe. Temperature limitations to preclude igniting any gas, dust, or fibers in the air, life testing to show robustness of wiring, spark and backfire emission tests, and precautions with fuel tanks all show that these rules dwell on fire and explosion safety.

National Fire Protection Association. The National Fire Protection Association (NFPA) has a standard on lift trucks (NFPA 505, 1996). The 1969 version of this standard is called out in the Code of Federal Regulations. The more recent 1996 version is discussed here because it is more up-to-date in dealing with contemporary lift truck safety issues. For example, the 1996 version has designations for compressed natural gas fired lift trucks, designators $\mathrm{CN}$ and $\mathrm{CNS}$, and G/CN or GS/CNS for dual fueled lift trucks that use gasoline and compressed natural gas. Often a manufacturer will perform a conversion to dual fuel in an effort to have the operator reduce operating costs. Lift trucks that have dual fuels must meet both the UL 558 standard and the NFPA Standard 52 (Standard for Compressed Natural Gas Vehicular Fuel Systems). If the lift truck is powered by gasoline and liquefied petroleum gas, then it must meet the requirements in UL 558 for both types of fuel.

NFPA 505 also defines the operations area Classes, Groups, and Divisions. It also outlines maintenance concerns; these have already been discussed in the Code of Federal Regulations. This standard cites adherence to other NFPA standards when dealing with fuel, such as NFPA 58 (Storage and Handling of Liquefied Petroleum Gases) and NFPA 30 (Flammable and Combustible Liquids Code). The standard is quite clear that any dents, scrapes, gouges to an LP gas tank that materially weaken its structure require the tank to be removed from service. There is no smoking in any refueling area to avoid possible ignition of fuel vapors (including hydrogen gas effluents from batteries being charged). The standard also specifies to add acid to water when replenishing battery electrolyte. Tools and other metallic objects shall be kept away from the tops of uncovered batteries (to avoid making a short circuit across the battery terminals). The standard also cited the American 
National Standards Institute (ANSI) standard B56.1 (Safety Standard for Low Lift and High Lift Trucks) as the operating rules for lift trucks.

American National Standards Institute. ANSI has a set of standards for lift trucks that deal with a variety of issues. The first standard to consider is ANSI/ASME B56.11993, Safety Standard for Low Lift and High Lift Trucks (ANSI, 1993). This standard offers more practical information about lift truck operations. This standard discusses stopping distances for stopping lift trucks on grades, the stability of lift trucks, fuel handling and storage, appropriate aisle spacing, lighting in the facility, control of noxious gases and fumes from lift truck exhausts, the sound emitted from the lift truck, deckboards (also called dockboards or bridge plates), warning devices (i.e., a horn and turn signals if needed), and there are instructions to follow if a lift truck is to be used to elevate people. Operator training is discussed in detail.

Some general safety rules are to start the lift truck only from the driver's seat, to keep hands and feet inside the lift truck while operating it, never put any body part between the mast and the truck, do not drive the truck up to anyone standing in front of an object, ensure personnel are clear of the rear swing area before conducting any maneuvers, safeguard pedestrians at all times, exercise care at intersections, cross aisles, doorways, or other locations where pedestrians might step out into the truck's path, do not allow anyone to stand under the elevated load of a lift truck, and do not allow passengers on the truck if it is not designed to seat them.

When using lift trucks in facilities, there are a set of ANSI operating rules. These include observing plant speed limits, maintaining a safe distance between vehicles, yielding the right of way to pedestrians, not passing another lift truck traveling in the same direction at intersections, blind spots, or other dangerous locations, crossing railroad tracks at an angle whenever possible, and keep a clear view of the path of travel (trail the load if necessary to achieve this view). Ascend and descend slowly. When ascending or descending a grade over 5\%, rider trucks shall be driven with the load upgrade to enhance truck stability. Unloaded trucks shall be operated on all grades with the load engaging means (i.e., the forks) downgrade to enhance truck stability. Several of these rules are also found in the OSHA discussion above.

Indications of excessive speed being applied during turns are tire skidding, lift truck side sway, wheel lift, and the need of the driver to grip the steering wheel tightly to keep from sliding out of the seat. The instructions are that the operator should ride with the lift truck if it tips over, and the operator should lean away from the point of impact (either falling off a dock or tipping on the floor of the facility). This rule is to protect the operator from being injured by the lift truck falling object protection system if the operator tries to leap from the cab during a fall. Leaping from the cab is the most natural reaction, but often there is not enough time for the operator to get clear before the vehicle impacts the ground. In Chapter 3 there are some case histories that show the operator cannot always get clear of a tipping vehicle. 
Rules for fueling are the same as those in the Code of Federal Regulations. Another section discusses the condition of the forks. The straightness of the forks shall be checked, both the upper face of the fork blade and the front face of the shank. If the deviation from straightness exceeds $0.5 \%$ of the length of the blade and/or the height of the shank, the fork must be retired from service until it can be repaired. If the difference in the fork tip height is greater than $3 \%$ of the length of the blade, the set of forks shall not be used until repaired. If the fork blade or shank are worn, with a special check at the heel of the fork, down to $90 \%$ of the original thickness, they shall be removed from service. Fork safety is also discussed by Crosby (1979), Martin (1981), and Schwind (1995). Some of the reasons for these rules about forks are discussed in these references. A $10 \%$ reduction in fork blade thickness results in a load weight capacity reduction of $20 \%$. Fork inspection frequency is suggested to be yearly. Since drivers are taught to drive with the forks low and parallel with the travel surface, many drivers will travel with the forks (especially the heels) riding on the ground. That practice causes heel abrasion. There is also abrasion and wear on the floor surface during normal operation, as well as contact wear by sliding the forks into and out of pallets (Schwind, 1995).

Fork repairs can only be performed by manufacturers or by experts of equal competence, according to the ANSI standard. Other repairs are also discussed. Hydraulic systems must be inspected regularly and maintained in conformance with good practice. These systems should be checked to ensure that hydraulic drift, or leakage of fluid has not developed to the extent that it creates a hazard. Lift trucks shall be kept clean to minimize fire hazards and facilitate detection of loose or defective parts.

Modifications and additions that affect the lift truck's capacity or safe operations shall not be carried out without the manufacturer's written approval. This is very important for lift trucks that have added features, such as barrel handlers, installed. A letter from the manufacturer is important to keep on file in the case that an OSHA inspector requests it.

Any replacement parts for lift trucks should be interchangeable with original equipment parts, or equal to original equipment. Indeed, when plant spaces are confined, any temporary replacement lift truck (to be used while the primary lift truck is undergoing repairs) should be the same model or at least comparable in its operating features. Features such as the turning radius, lift height, rating, and capacity are necessary to allow the temporary truck to function.

The ANSI standard also discusses manufacturer information to test lift truck models for tilting (a stability test to determine how easily a lift truck can tip over), for capacity, for steering and braking, and the overhead guard. The deformation of the overhead guard is the deflection distance when the reference weight is dropped on the guard. The reference weight begins at $340 \mathrm{~kg}$ ( 750 pounds) for a lift truck with a capacity of $1364 \mathrm{~kg}(3,000$ pounds), and increases with increasing capacity. A distance of $25.4 \mathrm{~cm}$ (10 inches) must remain from a horizontal line out from the tip of the steering wheel and the inside top of the guard after impact of the reference weight. Other safety information is also given in this standard. 
There are other standards in this ANSI series. ANSI B56.11.3 (1992) is a standard describing load handling symbols for levers and controls in lift trucks. The next standard for our consideration here is ANSI B56.11.4 (1992a). This standard defines safety ratings for hook-type forks and fork carriers. It establishes interchangeability for fork arms and fork carriers for lift trucks. The next ANSI standard is B56.11.5 (1992b), which specifies the test procedures and conditions for evaluating the noise generated by lift trucks. Lift truck operators wear microphones near their ears for this test. The microphones measure the sound pressure level in decibels on the A-weighted scale. Four test modes are required - mode 1 is a stationary truck lifting a load, mode 2 is a stationary truck idling at the manufacturer recommended idle speed, mode 3 is driving an unloaded lift truck at maximum travel speed, and mode 4 is driving a loaded lift truck at maximum travel speed. Another test requires taking the background noise reading at the facility, and then measuring the lift truck noise added to the existing environmental noise. The final ANSI standard is B56.11.6 (1992c). This standard gives test criteria and conditions for testing visibility from lift truck cabs. An array of lamps is placed at the location where the driver's head would be (above the seat index point) and light and shadow areas around the lift trick are observed. Acceptable visibility for traveling and maneuvering are based on measurements of dark shadows cast on screens placed around the lift truck. Because of the mast, fork carriage, and falling object protection system, forward visibility is not as clear in a lift truck as in other vehicles.

Society of Automotive Engineers. While the Society of Automotive Engineers (SAE) defers to the ANSI/ASME standards for powered industrial trucks (SAE, 1986), there are some useful guidance documents among the SAE collection. There are two tests outlined, one for backup alarms (SAE, 1993) and one for horns (SAE, 1989). The backup alarm test guidance does not select a sound level for the backup alarm, some alarms only generate one sound level $(120 \mathrm{~dB}-\mathrm{A})$, while others are adjustable. The horn guidance gives a range of horn sound levels, type $\mathrm{J}$ is $117 \mathrm{~dB}-\mathrm{A}$, type $\mathrm{K}$ is $112 \mathrm{~dB}-\mathrm{A}$, type $\mathrm{L}$ is $107 \mathrm{~dB}-\mathrm{A}$, type $\mathrm{M}$ is $97 \mathrm{~dB}-\mathrm{a}$, and type $\mathrm{N}$ is $87 \mathrm{~dB}-\mathrm{A}$, all of which should not vary by more than $\pm 4 \mathrm{~dB}-\mathrm{A}$ for normal system voltages. The standard does not specify which type of horn should be used in which location or facility. A rough rule of thumb mentioned by a lift truck sales firm is that backup alarms should be set at about 3 to $10 \mathrm{~dB}-\mathrm{A}$ higher sound level than the normal environment sound level.

A useful SAE information report (SAE, 1986a) discusses fire resistant hydraulic fluids. There are four types of these fluids. The types are HFA, a high water content oil; HFB, water-in-oil emulsion; HFC, glycol or polyglycol solutions; and HFD, anhydrous synthetic fluids. The system performance with these fluids is inferior to that with mineral oils, and derating the lift truck load capacity may be necessary to avoid undue component life limitations. Elastomer seals must be chosen with care when using fire resistant hydraulic fluids due to chemical compatibility issues. Another recommended practice (SAE, 1986b) discusses a classification system for hydraulic leaks in both clean and dirty operations. The classes for dirty operations are: class $O D$, no indications of moisture; class $1 D$, dry collection which does not propagate; class 2D, moist thin layer (less than $3 \mathrm{~mm}$ deep) of dust; class 3D, moist thick layer of dust (over $3 \mathrm{~mm}$ deep); class 4D, recurring fluid forms on vertical surfaces, dripping occurs, or pools of oil collect on the top of horizontal 
surfaces; and class 5D, recurring fluid where frequency of droplets makes a measurable stream of leakage.

There are several safety guides published by the SAE. The fire prevention information report (SAE, 1985) discusses using spark arrestors, minimizing any debris that could be flammable (accumulating debris and poor housekeeping are a factor in $45 \%$ of fires studied by the SAE), providing fire extinguishers and training operators on their use, and inspecting the machines to prevent component failures that could lead to fires (electrical insulation damage, fuel leaks, etc.). Two other standards for personnel protection (SAE, 1992; SAE, 1993a) offer some important advice. Hydraulic systems operate under enough pressure that leaking oil could penetrate the skin and cause serious injury or death. Use paper or cardboard to search for leaks, never use the hands to detect leaks. Other issues discussed are safe entry and egress from the vehicle, protection from rotating shafts and hot engine parts, and fire protection. The second standard discusses rollover protection and falling object protection. Seat belts for the operator are required for the roll over protective structure, but not for the falling object protective structure (FOPS). The FOPS should provide protection from objects such as trees or rocks, bricks, concrete blocks, and small hand tools that may fall from heights of not more than $9 \mathrm{~m}$ (30 feet) above the ground. Lift trucks are not required to have rollover protection, but must protect the operator from falling objects by an overhead guard (such as a FOPS) and also by a load backrest extension if the application warrants one (CFR, 1996). Another operator safety guide (SAE, 1987) is meant for tractors, but many of the suggestions are very applicable to lift trucks. Operators should not wear loose fitting clothing, long jewelry, or uncovered long hair, since these things can get caught in the machinery. Wear protective equipment, such as gloves or hearing protection. Wear the safety belt if it is provided. Use the steps and hand holds provided when climbing up and down, face the machine when climbing, do not jump off the machine. Maintenance practices are also described. There are refueling suggestions as well - do not smoke in the vicinity, do not fill a gasoline tank to the brim (leave ullage for expansion), and do not use gasoline as a solvent or for cleaning purposes.

Factory Mutual. This company has published some information and guidance on lift truck safety. They point out some of the financial losses that can occur through careless operation of forklifts. In the period between 1985-1989 in the US, lift trucks breaking fire sprinkler piping accounted for about a third of the monetary losses involving lift trucks (FM, 1991). There is also a concern about fires. Lift trucks themselves may not catch on fire, but they can ignite combustible materials in the facility. Also, lift trucks are often operated in severe service (frequent starts and stops, much time under heavy load). If the lift trucks are dirty and/or poorly maintained, they are a fire hazard. Fire extinguishers, to be carried on the internal combustion lift truck and at the refueling station, are recommended. Electric lift trucks have short circuits as a principal source of ignition. Most gasoline fueled lift trucks have fuel leakage as a primary source of fires, or spillage during refueling. There is little Factory Mutual data on diesel fueled lift truck fires. LP gas fueled lift trucks have had fires from breaks or leaks in the fuel lines, and from the relief valve lifting in areas of high heat (e.g., near furnaces, fire near fuel tank, or overfilled fuel tank). 
Another information source from Factory Mutual is their handbook (FM, 1967). While this handbook is dated, much of the information is still good advice. The handbook points out that lift truck damage to sprinklers, fire doors, and other fire protection devices that limit the spread of fires can increase the consequence of a fire. Mechanical damage to process equipment, piping, drums, or tanks could release flammable liquids or other flammable materials. Maintenance on lift trucks is important for their safety. Engine tuning, and cleaning with steam or alkaline solutions is recommended. Fire extinguishers are again recommended to be kept with the lift trucks. Other recommendations are similar to those in the loss prevention data sheet discussed above.

National Safety Council. There are several National Safety Council publications that address powered industrial trucks. Laing (1992) devotes a chapter to lift truck descriptions and safety issues. These issues include operator ergonomics, operator safety, and colocated worker safety. Another text (NSC, 1991) discusses good safety practices in battery recharging and refueling internal combustion engines, and a summary of rules from OSHA is also included. There is a section on byproducts from internal combustion engine exhausts and keeping the workplace air clean enough to meet OSHA standards for chemical inhalation exposure. Brodbeck (1996) also discusses some of the safety features to be found at road truck docks, where truckers interface with lift truck operators. Dock safety is important for avoiding collisions between workers and lift trucks.

Department of Energy. The US Department of Energy (DOE) has an occupational safety and health guide (DOE, 1994). The US DOE states that good prevention of lift truck accidents is proper maintenance, trained operators, and adherence to established safety procedures. A statement is made that accidents often occur when the lift truck driver parks the truck and leaves the immediate area; other employees needing passage attempt to move the lift truck. Other accidents involve carrying unstable loads, tipping over, dropping loads on the operator or others nearby, running into or running over pedestrians, or pinning people between trucks or between a truck and a fixed object. A meaningful training program for drivers is stressed as very useful in safe operations. Some safety equipment items that are not standard on lift trucks, but are cited in this guide consist of: wheel plates to protect people from objects picked up and thrown by tires, on-board fire extinguishers, and horns to warn others that a truck is moving forward.

The DOE hoisting and rigging manual (DOE STD-1090-96, 1996) contains a chapter on lift trucks. While it is only a standard, it states requirements for lift trucks, such as lift trucks shall be equipped with an operator-controlled horn or other signaling device, and users shall determine if blinkers or other visual warning devices are needed. If attachments are used on the forks, then the truck manufacturer must give a new truck load capacity and elevation rating. Modifications to a lift truck require written permission from the truck manufacturer. This standard also calls out compliance with NFPA 505, the CFR on lift trucks, UL 583 and UL 558, and the ASME B56 series. This standard also has an inspection checklist for the daily inspection and calls out periodic detailed inspections to be conducted every 6 months. There is a description of conduct of operations for lift truck drivers. A safe operating maximum speed limit inside buildings of $5 \mathrm{mph}$ is given. A section on proper techniques for lifting personnel with a lift truck is also given. The 
operations section stresses that the lift truck driver must obey the "stop" hand signal, no matter who gives the signal.

Conclusions. There are numerous rules, recommended practices, guidance, and consensus standards for powered industrial trucks. In general, the rules address hazards and safety concerns discussed in Chapter 3. The rules generally seem to place safety first without intentionally trying to impede productivity or convenience, although many would argue that these rules do impede productivity. Nonetheless, there are roughly 100 workplace fatalities related to lift trucks each year in the US. Perhaps the discussion by Geller (1996) about safety being a workplace priority, and priorities changing to meet the business needs of the workplace, is true in the case of lift trucks. Either these rules, practices, and guides are not comprehensive enough to decrease lift truck-related fatalities to nearly zero each year, or they are not being followed because priorities have changed to meet economic and other business pressures. The rules appear to be very comprehensive. OSHA has proposed a new operator training rule in the Federal Register to address the continued workplace fatalities, this rule is discussed in the next chapter on selected safety issues.

\section{Chapter 4 References}

ANSI, 1992.

ANSI, 1992a.

ANSI, $1992 b$.

ANSI, 1992c.

ANSI, 1993.
American National Standards Institute/American Society of Mechanical Engineers, Load Handling Symbols for Powered Industrial Trucks, ANSI/ASME B56.11.3-1992, revision of 1988 edition, 1992.

American National Standards Institute/American Society of Mechanical Engineers, Hook-Type Forks and Fork Carriers for Powered Industrial Forklift Trucks, ANSI/ASME B56.11.4-1992, revision of 1988 edition, 1992.

American National Standards Institute/American Society of Mechanical Engineers, Measurements of Sound Emitted by Low Lift, High Lift, and Rough Terrain Powered Industrial Trucks, ANSI/ASME B56.11.5-1992, revision of 1989 edition, 1992.

American National Standards Institute/American Society of Mechanical Engineers, Evaluation of Visibility from Powered Industrial Trucks, ANSI/ASME B56.11.6-1992, 1992.

American National Standards Institute/American Society of Mechanical Engineers, Safety Standard for Low Lift and High Lift Trucks, ANSI/ASME B56.1-1993, revision of 1988 edition, 1993. 
Brodbeck, 1996.

CFR, 1996.

CFRa, 1996.

Crosby, 1979.

DOE, 1994.

DOE STD-1090-96, 1996.

Evans, 1979.

FM, 1967.

FM, 1991.

Geller, 1996.

Laing, 1992.

Martin, 1981.
J. E. Brodbeck, editor, Motor Fleet Safety Manual, fourth edition, National Safety Council, Itasca, Illinois, ISBN 087912-188-2, 1996, Chapter 7.

US Code of Federal Regulations, Title 29, Labor, Section 1910.187, Powered Industrial Trucks, July 1, 1996.

US Code of Federal Regulations, Title 29, Labor, Section 1926.602, Material Handling Equipment, (c) lifting and hauling equipment, July 1, 1996.

J. R. Crosby, "Fork Failure -- It Can Be Avoided," Industrial Engineering, 11, June 1979, pages 40-43.

DOE Occupational Safety and Health Technical Reference Manual, published by Government Institutes, Inc., Rockville, Maryland, ISBN 0-86587-396-8, April 1994, Chapter 6.

US Department of Energy Standard, Hoisting and Rigging, DOE STD-1090-96, September 1996, chapter 10.

D. W. Evans, "A Brief Rundown on Current Lift Truck Safety Requirements," Plant Engineering, 33, October 31, 1979, pages 59-61.

Factory Mutual Engineering Corporation, Handbook of Industrial Loss Prevention. recommended practices for the protection of property and processes against damage by fire. explosion, lightning, wind, earthquake, second edition, McGraw-Hill, New York, 1967, Chapter 67.

Factory Mutual Engineering Corporation, Loss Prevention Data sheet 7-39, Industrial Trucks, February 1991.

E. S. Geller, The Psychology of Safety, how to improve behaviors and attitudes on the job, Chilton Book Company, Radnor, Pennsylvania, ISBN 0-8019-8733-4, 1996, Chapter 1.

P. M. Laing, editor, Accident Prevention Manual for Business and Industry. engineering and technology volume, tenth edition, National Safety Council, Itasca, Illinois, ISBN 0-87912-156-4, 1992, Chapter 11.

C. H. Martin, "How to Check Lift Forks for Safety," Plant Engineering, 35, May 1981, pages 219-221. 
Mruk, 1973.

NFPA 505, 1996.

NSC, 1991.

SAE, 1985.

SAE, 1986.

SAE, 1986a.

SAE, 1986b.

SAE, 1987.

SAE, 1989.

SAE, 1992.

SAE, 1993.

SAE, 1993a.
J. Mruk, "How Underwriters' Laboratories goes about Testing Industrial Trucks for OSHA Compliance," Plant Engineering, 27, July 12, 1973, pages 102-105.

National Fire Protection Association, Fire Safety Standard for Powered Industrial Trucks Including Type Designations. Areas of Use, Conversions, Maintenance, and Operation, 1996 edition, NFPA 505, NFPA, Quincy, MA, 1996.

National Safety Council, Supervisor's Safety Manual, 7 th edition, NSC, Itasca, Illinois, 1991, pages 205-210.

Fire Prevention on Forestry Equipment, information report, SAE J1212, Society of Automotive Engineers, January 1985 .

Categories of Off-Road Self-Propelled Work Machines, recommended practice, SAE J1116, Society of Automotive Engineers, June 1986.

Fire-Resistant Fluid Usage in Hydraulic Systems of OffRoad Work Machines, information report, SAE J1447, Society of Automotive Engineers, May 1986.

External Leakage Classifications for Hydraulic Systems, recommended practice, SAE J1176, Society of Automotive Engineers, March 1986.

Operator Precautions, recommended practice, SAE J153, Society of Automotive Engineers, May 1987.

Horn - Forward Warning - Electric - Performance, Test, and Application, recommended practice, SAE J1105, Society of Automotive Engineers, September 1989.

Personnel Protection for General Purpose Industrial Machines, standard, SAE J98, Society of Automotive Engineers, November 1992.

Alarm - Backup - Electric Laboratory Performance Testing, standard, SAE J994, Society of Automotive Engineers, August 1993.

Operator Protection for General Purpose Industrial Machines, standard, SAE J1042, Society of Automotive Engineers, June 1993. 
Schwind, 1995.

G. F. Schwind, "Lift Truck Forks: Maintenance and Inspection," Material Handling Engineering, 50, March 1995, pages 28-29.

UL 558, 1991.

Underwriters Laboratories, Standard for Industrial Trucks, Internal Combustion Engine-Powered, UL 558, eighth edition, August 6, 1991.

UL 583, 1991.

Underwriters Laboratories, Standard for Electric-BatteryPowered Industrial Trucks, UL 583, eighth edition, September 26, 1991. 


\section{Current Issues and Concerns for Lift Truck Safety}

This chapter contains a discussion about several current safety issues and ideas regarding lift trucks. There are several current ideas on safety for lift trucks. These are: seat belts or restraints for drivers, fire extinguishers aboard the lift truck, engine fire suppression system, driver visibility, traffic safety, training, ergonomics, and add-ons to trucks (to tines, etc.). Each of these topics are addressed below.

Seat belts. As seen in Chapter 4, there are no rules that require the use of seat belts for forklift trucks. Seat belts are suggested by several organizations, so that in the event of a tip over or a lift truck fall (as in from a dock) the driver rides with the lift truck instead of trying to get clear - which usually results in the driver being trapped and possibly crushed by the structure of the FOPS. Unfortunately, drivers of lift trucks are not like road truck drivers, who have long sedentary times of driving each workday. Lift truck drivers must climb onto and off of their vehicles many times each day. They must check manifests and orders of what to move where, they may need to disembark to read wording on loads, they may need to open and close road truck doors or rail car doors, they may have to adjust loads or tie them down, they may need to open and close building doors, and other duties that must be performed. With the driver leaving the vehicle from time to time, the seat belt can be a nuisance. Impractical safety equipment is often not used. Therefore, perhaps there are other means to protect the driver without using seat belts. Perhaps a seat with wrap-around arm rests to keep the driver stable when the truck tilts. The seat could be detent swiveled (i.e., faces front or faces $90^{\circ}$ to the left or right) to allow the driver to get in and out of the lift truck easily, but would keep the driver away from the edges of the lift truck if the truck were to fall. The ANSI standard in the previous chapter described that the best procedure for lift truck drivers is to ride out with the lift truck if it tips or falls, and keep away from the impact point [presumably to keep limbs from being pinched or shattered between the truck and the ground]. This seat design could help the driver to do the safer behavior in case of an accident and it could help the driver with routine boarding and egress from the vehicle.

Fire extinguishers. There are no rules about lift trucks being equipped with fire extinguishers, although some NFPA and DOE guidance recommends on-board fire extinguishers. Perhaps if the company in question has adopted the National Fire Protection Association's Life Safety Code (NFPA 101, 1994), which states that personnel must be within a specified distance (an example is $15 \mathrm{~m}$, or 50 feet) of fire extinguishers. If the lift truck driver is the only person in a given area of the facility at a time, then the driver must be close to a fire extinguisher. Depending on the size of the facility, it might be easier and cheaper for one fire extinguisher to be mounted on the lift truck than several mounted at various areas around the facility. If the driver is not alone in the facility, then it is probably easier to have stationary fire extinguishers mounted in routine locations. The lift truck fire safety record is sufficiently good (see the next chapter) that no fire extinguisher appears to be necessary for the lift truck units themselves. Frequencies are discussed in the next chapter, but lift trucks in general have lower fire frequencies than automobiles (e.g., less than about 1E-03/year) and automobiles do not have mandatory fire extinguishers.

Engine fire suppression systems. There are no rules that require lift trucks to have an in-vehicle fire suppression system similar to large construction equipment. While lift trucks have had fires, these have been relatively few. As discussed above, automobiles do not have engine fire suppression systems because an engine kept in proper working order (good hoses and seals) and kept clean (no grime or oil buildup, no foreign material buildup) will not be likely to catch fire. A fire suppression system will add weight to a vehicle, it will increase the maintenance cost and outage time for inspectors to verify 
functionality on a regular basis (perhaps yearly), and add to the capital cost of the lift truck. An engine fire suppression system might be carbon dioxide or perhaps a halogenated agent flooded into the engine compartment. A cost benefit analysis should indicate that the expense of this mitigative system is not warranted due to the low frequency of the lift truck fire, unless the consequences of such a fire are catastrophic. If the consequences (smoke damage, process interruption, etc.) are very large, then an engine fire suppression system would need further investigation.

Driver visibility. Lift trucks are noted for their poor visibility. Drivers learn to adjust to the blocked vision. They learn to look around the mast, the hydraulic system, and the falling object protective structure posts. There is a visibility test for lift trucks, as discussed in Chapter 4. Other ideas for lift truck visibility safety are to make areas of the facility into lift truck exclusion areas, or safe areas, so that the pedestrians know that there is an area free of lift trucks. Segregating the pathways can help with lift truck-pedestrian accidents, but it has been found to be difficult to enforce. Another feature noted in the literature (Bests, 1988) was changing the color panel of the lenses on warning strobes mounted on the lift truck falling object protective system. The idea is that if the driver cannot see workers very easily, then make the lift truck more easily visible to the workers. Every week, or some other time interval, the safety personnel can change the plastic panel on the lift truck flashing visual signal, so that each week workers must get used to a different color (blue, red, amber, etc.). This rotation of colors helps to maintain the worker's awareness of lift trucks. Another add-on would be mirrors for the driver to use when operating the lift truck. The facility can also use hemisphere mirrors mounted on the ceiling at intersections, so the driver is alerted to oncoming cross traffic.

Traffic safety. Several authors cited in this report noted that facility layouts needed to consider safety rules developed for highway traffic (Booth, 1979; O'Mara, 1989). The US Code of Federal Regulations states that drivers shall follow plant speed limits, and the fact that lift trucks only travel at slow speeds (usually under $16 \mathrm{kph}$, or $10 \mathrm{mph}$ ) should promote a safe situation. However, some facility or plant layouts are not very good because they mix or cross pedestrian and vehicle traffic. Some excerpts from lift truck driver statements are enlightening to show their concern and apprehension in interacting with unprotected workers (Larsson and Rechnitzer, 1994):

"space is too confining around those machines - there is always a risk of hitting someone or driving over someones toes"

"anyone might step out from between those stacks and I would not be able to stop"

"if someone is standing there drinking water when I come to pick up my order, I almost nudge his behind with the mast"

"even if I manage to stop the forklift, the load will continue off the tines and hit the person"

"just before the end of the shift, the operators run like rabbits all over the place, it's not safe to use the forklifts at that time" 
Traffic safety is important for personnel safety in the plant building. In Chapter 3 there were some examples of injuries obtained from lift truck collisions with pedestrians. There are some safety rules given in the Code of Federal Regulations on lift truck driving safety (keep within speed limits, keep reasonable distance between lift trucks, yield right of way, etc.). There are some more guidelines given by the National Safety Council (NSC, 1992).

No written rules on speed limits have been found. This issue is apparently left to be determined for each individual plant site. Authors recommend walking speed as a speed limit inside buildings (that is, 3 to $4 \mathrm{mph}$ ) (for example, see Lovested, 1977). The US DOE gives an upper limit of $5 \mathrm{mph}$ inside buildings in its lift truck guidance (DOE, 1996). Since lift trucks are not usually equipped with speedometers, an inexpensive method to test lift truck speeds is pacing off a distance and using a stop watch to time the lift truck as it traverses the distance (perhaps without telling the drivers that they are being monitored), then converting the feet traveled per second into an hourly speed. For example, 4.4 feet per second is $3.5 \mathrm{mph}$.

Operator training. Providing effective training for operators of lift trucks has become a more and more important priority for companies. Training usually begins with verifying that an operator has an automobile driving license, since this license has already determined vision requirements and any other special restrictions or requirements. The original lift truck training manual (NIOSH, 1978) is a basic guide and a useful publication, but many industry personnel (for example, see Schwind, 1990, and Swartz, 1993) believe that training can be taken much further than a classroom lecture based on that manual.

In a behavioral based study process (WSA, 1982), lift truck operators were observed while following their normal routines, then they were trained on safety. Next, the operators received follow-up checks, feedback, and supplemental training when needed. This is a considerable investiture of time and resources, but there is the offset that the facility will not pay as many workman's compensation claims when there are fewer worker injuries. The WSA study at a warehouse showed these error rate per operation results:

Operator Behavior Pre-training average error rate $\quad$ Retention average error rate
warns other lift trucks
0.18
0.04
yields to other lift trucks
0.28
0.11
warns coworkers
0.64
0.13
sounds horn at blind
0.31
0.06
intersections
slows down at blind
0.63
0.25
intersections
looks at blind intersections
0.42
0.13 
Operator Behavior Pre-training average error rate

looks in direction of travel

0.30

0.24

0.14

avoids quick starts and abrupt

changes of direction $\underline{\text { Retention average error rate }}$

0.11

0.09

0.03

0.02

keeps all body parts in lift truck

0.15

0.07

maintains forks in proper position

0.16

maintains balanced load

0.12

0.04

drives in reverse when needed

0.17
0.03

From these results, it is easy to see that the drivers began behaving much more safely than before the training was attempted. In fact, behavior based training is advocated in many areas, not just lift truck driver training (Geller, 1996). The behavior based training is successful, which is also referred to in the Federal Register discussion on training (FR, 1995). The proposed changes to operator training are to adopt the training guidance in the consensus standard on powered industrial trucks (ANSI, 1993). There will also be a need to document training in some manner to verify that the training has been carried out. The proposed rule also includes two appendices. The first appendix provides guidance on setting up a training program and a rather comprehensive list of topics to cover in training, and the second appendix covers basic ideas of lift truck stability to avoid tipping the lift truck. Only time will tell if this rule is adopted, and if it is, what impact it has on future injury and fatality rates.

Ergonomics. There are several ergonomic issues with lift trucks. From Chapter 3, the foremost issue appears to be that drivers suffer many injuries embarking and disembarking from the lift truck. Design attention to hand and foot holds may help this problem. Another issue is the jarring and shock operators receive to their spines when the lift trucks travel over uneven ground. A change to consider is extra padding, suspension, or some form of low cost vibration dampening for the driver's seat (see Ozkaya et al., 1996). A third ergonomic issue is that the drivers must turn sideways in the seat to look to the rear of the vehicle, so that the drivers are looking in the direction of travel when the lift truck loads block forward view. Some other ergonomic features to help operators (NSC, 1992) are rear view mirrors, sound deadening material to reduce internal combustion engine noise, and turn signals to alert other lift truck drivers about the path being driven.

Add-ons. Additional equipment for lift trucks has been created to make some jobs easier. There are attachments for barrel handling, slip plates, carton stackers, bale clamps, and other specialized functions. Hunt (1981) discusses the common problems with 
attachments. The connection to the lift truck hydraulic system must be examined to verify that the lift truck system will be compatible with the attachment. If the hydraulic system does not deliver enough power to the attachment, it cannot function up to its capacity. Attachments can become contaminated as well, such as by 'pull-in' dirt on the hydraulic cylinder rod (Anders, 1983). Hunt suggests that when installing an attachment, flush the hydraulic oil through the lift truck filter for several minutes to remove any foreign materials, and change the filter regularly. If the load begins to drop, the problem is either loss of hydraulic fluid or an internal problem (worn cylinder packings or a faulty valve). If the attachment operates erratically, check for a flapper in the hydraulic line. A flapper is a small piece of rubber from the hose wall, formed from a cut or a nick. The flapper impedes flow, creating an action similar to that of a one-way intermittent check valve.

As discussed in Chapter 4, it can be important to get a letter from the attachment manufacturer that states the attachment can be used for the intended usage on the lift truck it is installed upon. A letter from the lift truck manufacturer stating the lift truck allowable load with the attachment is also needed. These letters will confirm any company actions about using the attachment if there is an inquiry by an Occupational Safety and Health Administration inspector.

\section{Chapter 5 References}

Anders, 1983.

ANSI, 1993.

Bests, 1988.

Booth, 1979.

DOE, 1996.
J. E. Anders, Sr., Industrial Hydraulics Troubleshooting, McGraw-Hill Book Company, New York, ISBN 0-07-001592-9, 1983, chapter 7.

American National Standards Institute/American Society of Mechanical Engineers, Safety Standard for Low Lift and High Lift Trucks, ANSI/ASME B56.1-1993, revision of 1988 edition, 1993.

Best's Safety Directory, volume II, "OSHA Requirements for Powered Industrial Trucks," A. M. Best Company, Oldwick, New Jersey, 1988 edition, pages 1141-1158.

R. T. Booth, "Making Factories Safe for Fork Lift Truck Drivers," Occupational Health, April 1979, pages 193197.

US Department of Energy Standard, Hoisting and Rigging, DOE STD-1090-96, September 1996, chapter 10. 
FR, 1995.

Geller, 1996.

Hunt, 1981.

Larsson and Rechnitzer, 1994.

Lovested, 1977.

NIOSH, 1978.

NFPA 101, 1994.

NSC, 1992.

O'Mara, 1989.

Ozkaya et al., 1996.
US Federal Register, Part II, Department of Labor, Occupational Safety and Health Administration, Powered Industrial Truck Operator Training: Proposed Rule, volume 60, number 49, Tuesday, March 14, 1995, pages 13782-13831.

E. S. Geller, The Psychology of Safety, how to improve behaviors and attitudes on the job, Chilton Book Company, Radnor, Pennsylvania, ISBN 0-8019-87334, 1996, Chapter 1.

R. L. Hunt, "Guidelines for Installing and Servicing Lift-Truck Attachments," Plant Engineering, 35, November 12, 1981, pages 118-121.

T. J. Larsson and G. Rechnitzer, "Forklift trucks analysis of severe and fatal occupational injuries, critical incidents and priorities for prevention," Safety Science, 17, 1994, pages 275-289.

G. E. Lovested, "Top Ten Fork-Lift Truck Accidents," National Safety News, 116, September 1977, pages 123-127.

National Institute for Occupational Safety and Health, Outline for Training of Powered Industrial Truck Operators, NIOSH publication number 78-199, October 1978.

National Fire Protection Association, Life Safety Code, NFPA 101, 1994 edition.

P. M. Laing, editor, Accident Prevention Manual for Business and Industry, engineering and technology volume, 10th edition, National Safety Council, Itasca, Illinois, ISBN 0-87912-156-4, 1992, Chapter 11.

N. O'Mara, A Study of Fork Lift Truck Accidents in South Australia, South Australian Health Commission, ISBN 0-7243-4027-0, February 1989.

N. Ozkaya et al., "Effect of Operator Seat Design on Vibration Exposure," American Industrial Hygiene Association Journal, 57, 1996, pages 837-842. 
Schwind, 1990.

G. F. Schwind, "Operator Training: More Than Just Driving a Lift Truck," Material Handling Engineering, June 1990, pages 32-40.

Swartz, 1993.

G. Swartz, "Forklift Safety Training, Tips for Improving Your Program," Professional Safety, 38, January 1993, pages 16-21.

WSA, 1982.

WSA, Inc., Long Term Effects of Learned Safety Skills, Demonstration of the Effectiveness of an Industrial Lift Truck Operator Safety Training Program Utilizing a Behavior Sampling Procedure, accession number PB83197012, WSA, Inc., San Diego, California, 1982. 


\section{Lift Truck Safety Analysis and Reliability}

This chapter contains information about lift trucks for safety analysis and reliability calculations. Lift truck safety analysis is performed when the lift truck handles hazardous materials, such as barrels of chemical or nuclear waste, or hazardous items (e.g., radioactively contaminated material during decommissioning, or chemical materials). A safety analysis for personnel injuries could be performed if there is a high number of events at a given facility. Generally, lift truck damage inflicted on loads (commercial products, non-building materials, hazardous chemicals, etc.) are not considered a threat to public safety - such damage is just an economic loss to the facility.

Lift truck safety analysis. There are several safety issues that a lift truck could create at a facility that handles nuclear materials or wastes. The major safety issues treated in the literature are, in general: lift truck catches fire, or the lift truck breaches a container by dropping or colliding with the container (see RWMC, 1996), although the hazards assessment in Chapter 3 can potentially provide more safety issues. Some other issues are discussed in other forklift safety reports, and these issues are discussed later in this section. Of the major safety issues, fire frequency is discussed first. While fires are apparently a rare event (recall in Chapter 3, there were 14 lift truck fires over 100 years in Australia), it must be studied, either to show that the frequency (and hence, the risk) is low or to show that consequences from a lift truck fire are tolerable. In a fire hazards analysis report for a waste repository (Davis et al., 1989) the frequency of a lift truck catching fire was given as 3.3E-05/year for an electric powered forklift. Internal combustion engine lift trucks are in this order of magnitude as well, as shown by Trusty et al. (1989) who discussed a propane forklift fire frequency of 4.7E-05/year for a 260 hour/year unit. These two values are within $50 \%$ of each other. Davis (1989) also reported a road vehicle fuel fire frequency of 1.6E-04/year for fossil fueled trucks such as boom trucks, bucket trucks, and road trucks. Another fire issue is the lift truck igniting a fire. Electric motor driven lift trucks can ignite fires without catching fire themselves; such as arcs that could ignite fires, as discussed in the classification system (E, EE, EX) defined in Chapter 4. Arcing is treated in Cobine (1941). There is a characteristic curve of gas breakdown voltage versus (air pressure)(air gap distance) for an electric arc to travel across a gap. This curve is called the Paschen curve. Since most electric lift truck voltage systems are 24, 36, or 48 Volts (Holzhauer, 1990), the voltage to force an arc across a gap is low, and the conductors would have to be nearly touching (perhaps at $0.1 \mathrm{~mm}$ distance or less) to allow an arc to propagate in air. A lift truck could emits sparks from the engine or from the exhaust system of internal combustion engine powered truck; , this must also be taken into account.

As a comparison to the fire frequency above, automobile fires have been reviewed. Automobile fires occur with a crude average frequency of 3.2E-03/year (SAUS, 1992; roughly 478,000 car fires in the year 1988 , about 147.5 million cars in the US in 1988). Butler (1986) gives 45,000 car fires in the United Kingdom, with 18,522,469 registered automobiles, or about $2.4 \mathrm{E}-03 /$ year as a crude average fire frequency. These two frequencies are within $33 \%$ of each other, which is good agreement for this type of estimate. However, there are several factors to consider in that frequency. About $50 \%$ to $60 \%$ of automobile fires are arson (Butler, 1986), but more recent work by Conley (1996) 
suggests that arson is only about $16 \%$ of yearly vehicle fires. Another point is that as automobiles age their fire risk increases (Cole, 1992). Butler (1986) states that a ten year old car's fire risk increases by a factor of 4 over that of a three year old car. The agerelated reasons are mainly deterioration of hoses and seals that allow leakage of combustible fluids on and around the engine. Sometimes these fluids can come in contact with very hot parts of the automobile and ignite. Therefore, the fire frequency is skewed toward older vehicles. Automobile usage and maintenance are not taken into account with this crude estimate, either. The lift truck fire frequency given above for lift trucks of indeterminate age is about an order of magnitude lower than the crude frequency for automobiles. This difference seems reasonable when one considers that lift trucks are required to be visually inspected daily, while civilian automobiles might be inspected by a mechanic perhaps once a year or less frequently.

The other safety issue is lift truck load drops or collisions. These events require treatment of operator error. One value of the basic human error rate for the operator performing poorly on the lift truck is 0.003 per demand (Trusty et al., 1989). For example, dropping a load, knocking over a drum as it is lifted off of a road truck, hitting a container with the forks while the container is on the loading platform, hitting a load with another load in transit, and other events. In work by Benhardt et al. (1994), the generic error rate for a lift truck operator dropping a load due to human error was obtained by polling several risk practitioners. The resulting error rates were $1 \mathrm{E}-03$ per operation (where operation was defined as lift, move, and lower the load) as the average, 1E-02 per operation for the high value, and a value of $1 \mathrm{E}-04 /$ operation for the lower bound case. There is no explanation for the factor of three difference in the two studies. Benhardt et al. continued on with their analysis and combined the generic value with site-specific data to give values of $5 \mathrm{E}-05$ per operation (error factor of 10) for the average, that is, hauling a typical load. The upper bound was $5 \mathrm{E}-04$ per operation (error factor of 10 ) for unusual, unevenly balanced loads, and the lower bound value was 1E-05/operation (error factor of 10) for standardized unit loads when a spotter is present to assist the lift truck driver. Benhardt also discussed puncturing a load with the forks of a forklift truck. Since they did not find any actuarial data from industry to use in a calculation, they made an engineering assumption that puncturing was as likely as dropping a load. The site-specific data was used as the starting point generic data for estimating a puncture event frequency. There had been three puncture events at Benhardt's site, so an adjustment in the site-specific data (3 punctures $/ 6$ drops) gave a puncture failure rate value of 3E-05/operation for the typical load, 3E-04 per operation for the unusual or imbalanced load, and 5E-06 per operation for the standardized unit load with a spotter present. All of the human error values given above have an error factor of 10. It is considered most appropriate to use the basic data rather than the modified data if the analyst is performing an analysis on another plant or facility.

In a safety analysis report about nuclear materials handling (PP, 1993), a lift truck accident involving a drum puncture is analyzed. In that event, a lift truck (unloaded) is traveling at a constant speed of $8 \mathrm{kph}(5 \mathrm{mph})$ and hits a drum. One of the forks punctures the drum, and the atmospheric pressure drum expels an amount of material commensurate with the displacement volume of the intruding fork. The material is modeled to leave the drum and form a spherically shaped concentration cloud in the air. The lift truck driver is assumed to 
have ten seconds to vacate the area, and the driver's inhalation dose is calculated based on these assumptions. The brief time given for evacuation is somewhat shorter than other times allowed in other safety studies, which are usually over one minute (see, for example, RWMC, 1996 which allows 2 minutes egress time).

In another report on safety in nuclear materials handling (Rountree et al., 1995), lift trucks and collisions are analyzed. A carbon steel drum (1.2 mm thick, or 0.0475 inches; 18 gauge) requires about 6400 pounds-force to make a lancing 2 -inch vee opening in the drum wall, and about 2300 pounds-force straight into the drum wall to tear an opening in a spearing fashion. Considering that an average weight for an unloaded electric lift truck is 4,000 to 6,000 pounds (Holzhauer, 1990), and deceleration distances on the order of a drum radius, there is ample energy in a moving lift truck for one or both forks to puncture a drum in a collision event. The work-energy theorem, (force)(distance) $=0.5$ (mass) (final velocity $^{2}$ - initial velocity ${ }^{2}$ ), shows that for a zero final velocity, 1.5 foot stopping distance and a 5,000 pound lift truck, the force is quite high. The American National Standards Institute (ANSI, 1991) gives information about steel drum construction details, such as wall thickness, material, etc.

Rountree et al. (1995) also consider other lift truck-related events concerning facility safety. These events are:

container breach or puncture

drop a loaded pallet

lift truck failure during transit operation with a full load

lift truck failure while positioning or raising a full load

lift truck failure while unloaded

personnel injury from lift truck-related event

removal of faulted lift truck for repairs

operator egress during an accident situation

The electric lift truck in Rountree's analysis operates about 250 hours per calendar year, and moves about $1200-1500$ pallets.

Other work on nuclear materials safety has generated lift truck data (Eisenhawer and Bott, 1996). Using incidents per load handled, these average values have been obtained:

transport incidents per load

general operations incidents per load

lift truck strikes, or drop incidents, per load
$1.2 \mathrm{E}-03$

$6.0 \mathrm{E}-04$

2.4E-04

The facility averages handling 6 loads per day in one shift operation. Since the loads are so sensitive, it is understandable that the work is performed carefully and with extra help (such as spotters, supervisors, fire watch, etc.).

In the industrial environment, the pace is much faster [a lift truck driver in industry will handle much more than 6 loads per day] and the consequences of an error are generally 
much lower. That is, at a warehouse or a wharf, there is no concern about initiating high explosive that surrounds a nuclear device. Taub (1988) gave an estimate that in an ideal industrial plant environment, a lift truck operator hits a structural member or piece of equipment (e.g., a rack or shelf, a door or door frame, another load, an electrical panel, a pipe, or a duct) about once a month. Rountree et al. (1995) discussed that lift truck brakes are usually on the drive wheels only, and that while these trucks move slowly, their weight makes for long stopping distances even with quick operator action. There is a brief discussion about stopping distances in Chapter 2.

Another safety analysis issue is the wooden pallets (ASME, 1989; ASME, 1987) that are used with lift trucks. Cote and Linville (1990) discuss idle pallets. As wooden pallets age, they dry out and begin to splinter. Stacks of aged pallets introduce a severe fire potential. Fine pieces of splintered wood can easily ignite from many sources (carelessly discarded cigarette, smoldering rags, etc.). Even when automatic sprinklers operate to control the fire, the undersides of the pallets remain dry and the fire can continue to spread.

Lift truck reliability. Rountree et al. (1995) developed failure rates for electric lift truck systems based on engineering judgment and discussions with a lift truck manufacturer. They chose to use a gamma distribution because it models increasing failure rates with time, and it is often used as a suitable life distribution model.

\begin{tabular}{|l|l|c|c|c|}
\hline \multicolumn{1}{|c|}{ Component } & Failure type & Failures/hour & $\begin{array}{c}\text { Shape } \\
\text { parameter }\end{array}$ & $\begin{array}{c}\text { Scale } \\
\text { parameter }\end{array}$ \\
\hline battery & wear out & $4.4 \mathrm{E}-07$ & 1.1 & $2.5 \mathrm{E}+06$ \\
\hline brakes & random failure & $4.3 \mathrm{E}-06$ & 1.0 & $2.3 \mathrm{E}+05$ \\
\hline $\begin{array}{l}\text { Mast/boom } \\
\text { hydraulics }\end{array}$ & wear out & $3.9 \mathrm{E}-05$ & 2.0 & $5.1 \mathrm{E}+04$ \\
\hline $\begin{array}{l}\text { Mast/boom } \\
\text { mechanics }\end{array}$ & wear out & $1.7 \mathrm{E}-06$ & 1.5 & $8.8 \mathrm{E}+05$ \\
\hline Speed control & random failure & $2.0 \mathrm{E}-07$ & 1.0 & $5.0 \mathrm{E}+06$ \\
\hline Wiring - cables & wear out & $3.0 \mathrm{E}-05$ & 1.1 & $3.7 \mathrm{E}+04$ \\
\hline $\begin{array}{l}\text { Wiring - short } \\
\text { to ground }\end{array}$ & random failure & $3.0 \mathrm{E}-06$ & 1.0 & $3.3 \mathrm{E}+05$ \\
\hline $\begin{array}{l}\text { Wiring - open } \\
\text { circuit }\end{array}$ & random failure & $3.0 \mathrm{E}-06$ & 1.0 & $3.3 \mathrm{E}+05$ \\
\hline $\begin{array}{l}\text { Wiring - short } \\
\text { to power }\end{array}$ & random failure & $1.0 \mathrm{E}-08$ & 1.0 & $1.0 \mathrm{E}+08$ \\
\hline $\begin{array}{l}\text { Wiring - } \\
\text { terminals }\end{array}$ & wear out & $5.0 \mathrm{E}-07$ & 1.1 & $2.2 \mathrm{E}+06$ \\
\hline
\end{tabular}

The failure rates can be used as (failure rate)(operating time) to obtain a failure probability over the time period if the operating time is small (for these failure rates, an operating time less than a few hundred hours). If the operating time is large, then the gamma distribution must be used to calculate a failure probability. Since these values are for an electric lift truck, only the brakes, hydraulics and mechanical equipment can be used for other lift 
trucks. Some other yearly failure probabilities (for a 1200 hour/year lift truck) given in Rountree et al. (1995) are:

Trip switch failure, two switches that both fail

4.5E-07

Safety stop fails

0.02

Brake temperature above hydraulic fluid ignition point

0.001

Lift truck brake failure

0.005

Lift truck fork/carriage detachment

$1 \mathrm{E}-04$

Hydraulic system failure (i.e., hose failure)

0.027

Hydraulic system failure and valve failure (drop load)

$7 \mathrm{E}-04$

Horn fails

0.038

Fork structural failure (5E-08/hour)

$6 \mathrm{E}-05$

Puncture drum with fork

$5 \mathrm{E}-04$

Internal combustion engine powered lift trucks are said to have useful service lives of 10 to 15 years, based on an average operation of 2000 hours/year, while the economic lifetime of the lift truck is about 5 years (Weiss, 1984) or seven years (Levan, 1980), with electric powered lift trucks being about a year longer. Outside of normal maintenance (engine oil change, engine tune up, air filter change, etc.), the engine and drive train should suffer no major failures over the economic lifetime of 10,000 operating hours, then the average failure rate can be expressed as a 50\% Chi-square distribution with no failures and 2 degrees of freedom, or $0.6931 /(10,000$ hours $) \approx 7 \mathrm{E}-05 /$ hour. An upper bound $95 \%$ failure rate would be $2.995 /(10,000$ hours) or about $3 \mathrm{E}-04 /$ hour (see Tobias and Trindade, 1995). At 12,000 hours, the electric lift truck motor and drive train has an average failure rate of $6 \mathrm{E}-05 /$ hour and an upper bound of about $2.5 \mathrm{E}-04 /$ hour.

Hydraulic hose failures are important for lift trucks. There are hazards with the hoses, as pointed out in Chapter 3. If a hose has a pin hole leak, the hydraulic fluid can jet out, making a hazard for personnel exposure. The fluid can also contact hot parts of the lift truck which can lead to fluid vaporization, ignition and fire. Hydraulic hoses have temperature limitations, usually about $200^{\circ} \mathrm{F}$ (Loder, 1982). Wacker (1989) discusses the failure modes of hydraulic hoses. The basic mechanisms by which hoses fail are pressure pulses or surges; high temperatures; chemical incompatibility with hydraulic fluid; hose routing that has bends, tension, or torque which degrade hose strength; and high fluid velocity in the hose. The hose failure rate above (hose breach, pressure loss that leads to hydraulic system failure) is an hourly failure rate of $2.3 \mathrm{E}-05 /$ hour. Comparing to military failure rate data for hydraulic hoses shows near equivalence; the generalized military value is about 2.9E-05/hour (NPRD, 1991).

Another reliability issue is the two chains that raise the fork carriage up and down as the hydraulic piston extends and retracts. The NPRD (1991) has an average failure rate for a timing chain of $8.9 \mathrm{E}-06 /$ hour. The chain used on lift trucks is built to be similar to a timing chain, so it would be reasonable to expect its failure rate to be close in value, although the lift truck chain does not ride on sprocket teeth, it rides in a channel or notch over the cross bar. As a conservatism, an upper bound failure rate of $1 \mathrm{E}-05 /$ hour can be used on lift truck chains. 
In a facility with multiple lift trucks, the availability of one truck is not an important issue, since there are other trucks available to perform tasks. Having a spare lift truck is a good practice to avert any productivity loss if there is a breakdown, but a spare may not be necessary. If the facility cannot economically tolerate a lift truck breakdown, and the Occupational Safety and Health Administration rules are clear that even small problems (burnt out light bulb, bad horn, etc.) will cause the lift truck to be removed from service for maintenance, then a spare truck is a wise idea. Levan (1980) suggests one spare for every 15 to 20 lift trucks in one shift operation. For three shift operation, the number of spares increases because the lift trucks wear faster than single shift units. From data given in Weiss (1984), an individual lift truck yearly availability should be on the order of $94 \%$ [(2000 hrs - roughly $120 \mathrm{hrs}$ maintenance $) \div 2000$ hour single shift work year].

Conclusions. This chapter has cited several studies and some data to assist the analyst if safety assessment or analysis of lift trucks is required for operational safety. Some reliability values for components of various types of lift trucks have been given as well.

\section{Chapter 6 References}

ANSI, 1991.

ASME, 1987.

ASME, 1989.

Benhardt et al., 1994.

Butler, 1986.

Cobine, 1941.
American National Standard for Materials Handling (Containers) - Steel Drums and Pails, ANSI MH2-1991, October 30, 1991.

American Society of Mechanical Engineers, Standard Procedures for Determination of Durability of Wooden Pallets and Related Structures, ASME/ANSI MH1.6-1987, 1987.

American Society of Mechanical Engineers, Definitions and Terminology Covering Pallets and Related Structures, ASME MH1.1.2-1989, 1989.

H. C. Benhardt et al., Savannah River Site Human Error Data Base Development for Nonreactor Nuclear Facilities, WSRC-TR-93-581, Westinghouse Savannah River Company, February 28, 1994.

J. A. Butler, "Motor Vehicle Fires in Ireland and the UK," Fire Prevention. Number 195, December 1986, pages 2225.

J. D. Cobine, Gaseous Conductors, theory and engineering applications, McGraw-Hill Book Company, New York, 1941, Chapter 7. 
Cole, 1992.

Conley, 1996.

Cote and Linville, 1990.

Davis et al., 1989.

Eisenhawer and Bott, 1996.

Holzhauer, 1990.

Levan, 1980.

Loder, 1982.

NPRD, 1991.

PP, 1993.

Rountree et al., 1995.
L. S. Cole, The Investigation of Motor Vehicle Fires, third edition, Cole Books, Novato, California, ISBN 0-93981822-1, 1992, page 98 .

C. J. Conley, "Deadlier Than You Think," NFPA Journal, 90, September/October 1996, pages 79-84.

A. E. Cote and J. L. Linville, Industrial Fire Hazards Handbook, National Fire Protection Association, Quincy, Massachusetts, ISBN 0-87765-368-0, 1990, Chapter 54.

M. L. Davis and D. G. Satterwhite, Fire Hazards Analysis of the Radioactive Waste Management Complex Air Support Buildings, EGG-WM-8703, EG\&G Idaho, Inc., Idaho National Engineering Laboratory, September 1989.

S. W. Eisenhawer and T. F. Bott, "Initiating-Event Frequencies for Nuclear Weapon Dismantlement Hazard Analysis," Proceedings of the International Topical Meeting on Probabilistic Safety Assessment (PSA '96), Park City, Utah, September 29 - October 3, 1996, American Nuclear Society, volume II, 1996, pages 977-982.

R. Holzhauer, "Lift Truck Specifications Chart," Plant Engineering, 44, February 8, 1990, pages 65-85.

H. Levan, "Lift Truck Maintenance Guidelines," Plant Engineering, 34, October 16, 1980, pages 123-126.

J. R. Loder, "An Introduction to Hydraulic Hose," Plant Engineering, 36, January 21, 1982, pages 147-149.

Nonelectronic Parts Reliability Data 1991, NPRD-91, Reliability Analysis Center, Rome, New York, May 1, 1991, page 2-77, 2-20.

Pantex Plant Final Safety Analysis Report. Zone 4 Magazines (Staging or Interim Storage for Nuclear Weapons and Components), DOE/AL/65030, unlimited distribution, US Department of Energy, April 1993, pages 7-35 to 7-41.

S. L. K. Rountree et al., Stage Right Operational Safety Analysis and Evaluation of Pantex Personnel Operations, SAND93-3920, Sandia National Laboratories, January 1995. 
RWMC, 1996.

SAUS, 1992.

Taub, 1988.
Radioactive Waste Management Complex Safety Analysis Report, INEL-94/0226, Revision 1, Idaho National Engineering Laboratory, effective November 4, 1996.

Statistical Abstract of the United States, 112th edition, U.S. Government Printing Office, ISBN 0-16-038080-4, 1992, pages 201 and 614, using 1988 estimates for the number of car fires and number of cars in the US.

R. S. Taub, "Protecting Structures and Equipment from Lift Truck Damage," Plant Engineering, 42, October 13, 1988, pages 112-114.

Tobias and Trindade, 1995. P. A. Tobias and D. C. Trindade, Applied Reliability, second edition, Van Nostrand Reinhold, New York, ISBN 0-442-00469-9, 1995, pages 70-72.

Trusty et al., 1989.

Wacker, 1989.

Weiss, 1984.
A. D. Trusty et al., Hazards Assessment of the Hazardous Waste Storage Facility, EGG-PRA-4032, EG\&G Idaho, Inc., Idaho National Engineering Laboratory, March 1989.

R. Wacker, "Hydraulic Hose Failure: Causes and Cures," Plant Engineering, 43, May 11, 1989, pages 66-68.

W. H. Weiss, "When Should Lift Trucks Be Replaced?," Plant Engineering, 38, August 23, 1984, pages 60-62. 